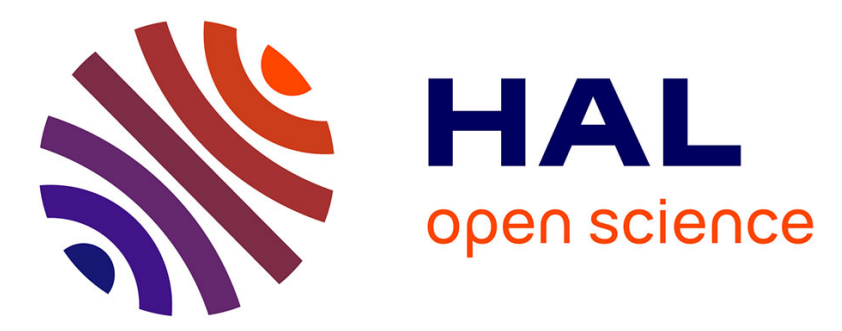

\title{
Sensitivity of convective structures to mean flow boundary conditions: A correlation between symmetry and dynamics
}

\author{
A Pocheau, François Daviaud
}

\section{- To cite this version:}

A Pocheau, François Daviaud. Sensitivity of convective structures to mean flow boundary conditions: A correlation between symmetry and dynamics. Biophysical Reviews and Letters, 1997, 55, pp.353373. 10.1103/PhysRevE.55.353 . cea-01373983

\section{HAL Id: cea-01373983 https://hal-cea.archives-ouvertes.fr/cea-01373983}

Submitted on 29 Sep 2016

HAL is a multi-disciplinary open access archive for the deposit and dissemination of scientific research documents, whether they are published or not. The documents may come from teaching and research institutions in France or abroad, or from public or private research centers.
L'archive ouverte pluridisciplinaire $\mathbf{H A L}$, est destinée au dépôt et à la diffusion de documents scientifiques de niveau recherche, publiés ou non, émanant des établissements d'enseignement et de recherche français ou étrangers, des laboratoires publics ou privés. 


\title{
Sensitivity of convective structures to mean flow boundary conditions: A correlation between symmetry and dynamics
}

\author{
A. Pocheau ${ }^{1}$ and F. Daviaud ${ }^{2}$ \\ ${ }^{1}$ Institut de Recherche sur les Phénomènes Hors-Equilibre, UMR 138 CNRS, Université d'Aix-Marseille I \& II, S 252, Centre de Saint \\ Jérome, 13397 Marseille, France \\ ${ }^{2}$ Service de Physique de l'Etat Condensé, CEA, Centre d'Etude de Saclay, 91191 Gif-sur-Yvette, France
}

(Received 20 June 1994; revised manuscript received 15 July 1996)

\begin{abstract}
Various simple structures have been proposed for modeling the transition to time dependence of convective patterns in extended geometries. In order to further question their relevance to the dynamics of complex structures (textures), we introduce a change of boundary conditions from both an experimental and a theoretical side. It consists in keeping the same roll structure but in separating the boundaries of the mean flows from those of the roll flows. This induces negligible effects on symmetric structures (straight rolls and foci) but dramatic changes on asymmetric ones (focus pairs and textures), especially regarding the onset of time dependence. Both kinds of sensitivity to this change of boundary conditions are recovered from the Cross-Newell equations. They reveal a correlation between symmetry and dynamics that prevents symmetric structures from modeling asymmetric ones. On the opposite side, they point to focus pairs as a plausible prototype of the mechanisms of time-dependence at work in textures. [S1063-651X(96)03912-8]
\end{abstract}

PACS number(s): 47.27.Cn, 47.20.Lz, 47.20.Bp

\section{INTRODUCTION}

Owing to nonlinear interactions between spatial modes, extended out-of-equilibrium systems provide fascinating but complex dynamics, still far from being understood. This has motivated a great deal of effort to model the interplay between their spatial and dynamical features [1]. The present work aims at improving the selection of such models in a well-controlled dissipative system: the Rayleigh-Bénard thermoconvection in moderate aspect ratio containers and small Prandtl number fluids.

In extended containers and close to the convective threshold, the convective structures generated without specific induction usually involve spatially disordered rolls showing curvature and defects [1]. However, in between defects, these so-called textures display much more ordered substructures. Their geometry, much simpler than those of textures, are close to those displayed by the following model structures: straight rolls, axisymmetrical rolls (hereafter called focus), two patches of curved rolls facing each other (hereafter called focus pair), and, in large aspect ratio containers, spiral rolls.

In moderately large containers, the behavior of model structures has been satisfactorily understood with a reasonable agreement between theories and experiments [1-3]. According to theories, important qualitative differences between model structures are in order however: infinite straight rolls provide large scale instabilities [4] and no intrinsic wavelength selection $[5,6]$ : axisymmetrical rolls provide both an intrinsic selection mechanism $[7,8]$ and a large-scale instability breaking their rotational symmetry $[9,10]$; focus pairs provide wavelength gradients and small-scale instabilities yielding the nucleation of propagating defects [11-13].

Owing to these qualitative distinctions, one might expect that the identification of the structure suitably modeling textures should be an easy task. This is not the case however for the following reasons. First, in moderate aspect ratios, experiments show that the symmetry breaking undergone by foci yields steady states whose routes to time dependence actually display features similar to those observed in focus pairs: wavelength gradients and small-scale instabilities [1419]. Second, both foci and focus pairs exhibit at any Prandtl number almost the same onsets for time dependence, at values similar to those displayed by textures [20-24]. From the experimental side, both the qualitative and quantitative features of these model structures are thus actually so close that it is not possible to decide which of them captures the mechanisms responsible for texture behavior.

In order to improve the study of model structures and their comparison with textures, we propose to modify the boundary conditions applied to convective structures. The change consists in separating the boundaries relevant to the primary roll flows from those relevant to the secondary mean flows by translating the latter into the conductive domain. This, applied to focus pairs, has already revealed a large inhibition of time dependence through an increase of their onset by a factor of ten [25]. The purpose of the present study consists in generalizing this change of configuration to all model structures and to textures.

Two different classes of behaviors are found depending on the structure: one involving a negligible change of the onset of time dependence and the other a spectacularly large one. The first class includes straight rolls and foci; the latter contains focus pairs and textures. These quite different sensitivities to a change of boundary conditions show that foci and focus pairs are not physically equivalent. Furthermore, for the present moderate aspect ratio container and small Prandtl number, texture behaviors appear compatible with a modelization by focus pairs but incompatible with a modelization by foci.

The respective origins of the two different classes are identified by analytically studying model structures. They reveal an essential role of asymmetric spatial distortions, what- 
ever their magnitude, in this convective system.

The paper is organized as follows. Section II introduces the so-called "open containers" in which the boundary conditions are implemented. The experimental results and the theoretical analysis are presented in Secs. III and IV, respectively. Their consequences are drawn in Sec. V and the conclusion of the study is reported in Sec. VI.

\section{OPEN CONTAINERS}

The principle of open containers is based on the secondary mean flows generated by convection in extended geometries. We recall their relevance to pattern dynamics in Sec. II A before addressing the definition and the main features of open containers.

\section{A. Mean flows}

Apart from other nonvariational effects, an important phenomenon breaking variationality has been pointed out by Siggia and Zippelius on the Boussinesq equations [26]. It consists of mean flows spontaneously produced, at finite Prandtl number, by unbalanced Reynolds stresses, the roll flows playing the role of anisotropic fluctuations.

Usually, these flows result from roll distortion and have a scale large compared to the roll width. They have been evidenced by tracer advection on asymmetric foci [16]. They interact with rolls by an advection forcing that may end in new pattern instabilities [29,9-13], wave-number gradients $[28,12]$, and time dependence [9-13]. They also induce nonlocality, first because, as any incompressible flow, they are nonlocally related to their sources and, second, because their advection forcing generates nonlocal interactions between rolls. All the theories proposed for model structures actually rely on them $[9-13,27]$.

\section{B. Definition of open containers}

Since both convective flows and mean flows are involved in convective structures, it makes sense dealing with their respective boundaries. We denote by "closed" containers the usual containers where the boundaries for mean flows and for convective flows are located at the same place. They are simply achieved by enclosing the convective domain by a rigid wall.

In contrast, we define as "open" containers the containers in which these boundaries are distant from each other. Since convection is a source of mean flow at finite Prandtl number, the only achievable configuration in practice corresponds to a mean flow boundary located outside the convective domain. This gives rise to three different regions: an inner convective zone, an outer conductive zone, and an interface in-between [Fig. 1(a)].

The main difference between these domains traces back to the potential or rotational nature of mean flows. Since the self-advection of mean flows is negligible (see Appendix A), the mean vertical vorticity only results from a balance between diffusion and forcing by mean Reynolds stresses. However, in both the convective and the conductive domains, the horizontal scale of variations of the relevant fields is so large compared to the cell depth that the vertical diffusion dominates the horizontal diffusion. Mean vertical vor-

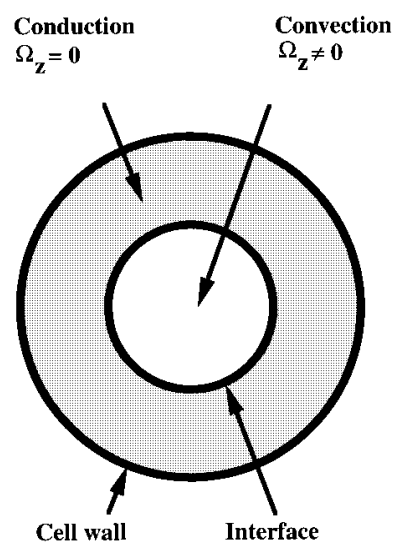

(a)

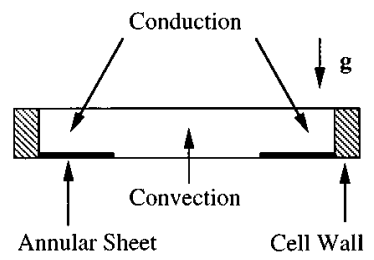

(b)

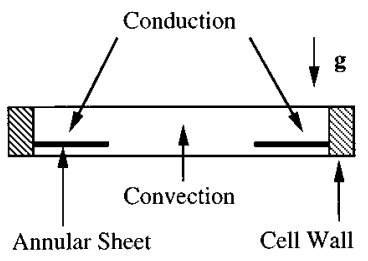

(c)

FIG. 1. Sketch of open containers. (a) The boundaries of roll flow and mean flow differ; three different domains may be defined according to the vanishing of convection, mean flow vorticity $\Omega$, or none, (b) [(c)] The conductive domain is forced by inserting a thin sheet that reduces the cell depth with minor consequences for the mean flow. When the sheet is in close contact with (at some distance from) the bottom plate, rolls tangential (normal) to boundaries are stabilized.

ticity is then directly linked, at each location, to mean Reynolds stresses, and thus vanishes in the conductive domain. Mean flows are therefore rotational in the inner zone and potential in the outer zone [Fig. 1(a)]. Their nature within the interface is addressed in Sec. IV A 2.

\section{Realization}

Realizing open containers requires annihilating the roll flow in an outer zone while preserving the mean flow. Taking advantage of the sensitivity of the Rayleigh number Ra to the cell depth $d, \operatorname{Ra} \propto d^{3}$, and of our proximity to the convective threshold, this selective action is obtained by slightly reducing $d$ in a definite part of the cell. The small channel reduction then produces subcritical conditions suppressing convection but yields minor modifications on mean flows (see Appendix D). In this configuration, the cell domain thus splits into a convective domain of unreduced depth $d$ and a conductive domain of reduced depth $d^{\prime}$. The roll flow 
boundary corresponds to the limit of the convective domain and the mean flow boundary is located, as usual, at the lateral walls of the cell [Fig. 1(a)].

In practice, the reduction of the cell depth has been achieved by inserting a thin sheet of cardboard at some definite places of a normal cell [Figs. 1(b) and 1(c)]. Its position with respect to the bottom plate determines the roll boundary condition: in the case of a close contact [Fig. 1(b)], rolls tangential to the sheet boundary are expected; on the opposite case [Fig. 1(c)], the usual situation corresponding to rolls perpendicular to the boundary is recovered.

When contact between sheet and plate is avoided, the sheet is placed at a distance $d_{1}$ of the bottom plate. Neglecting its thickness with respect to the cell depth $d$, we note $d_{2}=d-d_{1}$ its distance to the top plate. We chose $d_{1}=d / 4$ and $d_{2}=3 d / 4$ in order to provide a large depth available to mean flows. Since the vertical temperature gradient is uniform, the threshold of convection is increased by a factor $\left(d / d_{2}\right)^{4}=(4 / 3)^{4}$ in the conductive domain compared to its value in the convective one. Moreover, denoting by $\mathrm{Ra}$ the Rayleigh number and $\mathrm{Ra}_{c}$ its value at onset of convection, the reduced Rayleigh numbers in the convective domain $\varepsilon=\left(\mathrm{Ra}-\mathrm{Ra}_{c}\right) / \mathrm{Ra}_{c}$ and in the conductive one $\varepsilon^{\prime}=\left(\mathrm{Ra}^{\prime}-\mathrm{Ra}_{c}^{\prime}\right) / \mathrm{Ra}_{c}^{\prime}$ are related by $\varepsilon^{\prime}+1=\left(d_{2} / d\right)^{4}(\varepsilon+1)$. No rolls can thus appear in the conductive domain until $\varepsilon \approx 2.16$. In addition, for higher values of $\varepsilon$, the roll amplitude $A^{\prime}$ in the conductive domain is weakened compared to its value $A$ in the convective domain in a ratio $A^{\prime} / A=\left(\varepsilon^{\prime} / \varepsilon\right)^{1 / 2}$, smaller than 0.3 until $\varepsilon=3$.

\section{Validation}

Since our study aims at clarifying intrinsic mechanisms of pattern dynamics, one must first ensure that the trick used to realize open containers does not modify pattern behaviors for a different cause than a change of mean flow boundary conditions.

Apart from the expected hydrodynamical influence, the sheet could modify convection by a thermal mean. Especially, owing to the large thermal conductivity of cardboard compared to the convective fluid, here a gas, the heat current flowing within the plexiglass sidewall could be derived through the sheet well inside the cell and then modify temperature fields even at the border of the convective domain. To prevent this effect, the cardboard sheet has not been attached to the sidewalls so as to cut the heat flow coming from it. In addition, its thickness $\delta$ was reduced to a small fraction of the cell depth $(\delta=d / 8)$ so as to minimize horizontal heat transport. Its length $l$ was then sufficiently large compared to its thickness $(l / \delta \approx 100)$ for ensuring a good thermalization with the gas and thus a large reduction of the thermal perturbation brought about by the sidewalls. Altogether, these conditions have likely produced less thermal perturbations than in the weakly forcing configuration studied by Ahlers and co-workers in which a thicker $(\delta=d / 3)$ and narrower $(l / \delta \approx 10)$ spacer tab attached to the sidewall was used [19].

In order to experimentally control the influence of thermal perturbations on dynamics, we have studied the route to time dependence in a circular open container displaying a conductive zone so narrow that hydrodynamics could only be neg- ligibly perturbed: convective zone radius $R=12.5 d$, cell radius $R^{\prime}=1.1 R$, conductive zone extension $R^{\prime}-R=1.25 d$. The only remaining influence could therefore only arise from thermics. However, as expected, no modification, either qualitative or quantitative, has been noticed with respect to a closed circular container of same aspect ratio $R$.

\section{E. Roll boundary condition}

Although the sheet has a passive role with respect to pattern behavior, it actually provides a new roll boundary condition that we clarify in the following. When the sheet, whatever its size, is placed in close contact with the bottom plate, it enhances the inhomogeneity of thermal conductivity and thus induces horizontal thermal gradients. Rolls are then expected to end tangentially to the boundaries, as confirmed by experiment in Sec. III C.

When the sheet is placed in between the fluid layer, it imposes an additional rigid boundary condition at a quarter of the cell depth. Since the fundamental mode of convection involves nodes at the upper and lower plates only, it cannot satisfy this condition and therefore vanishes at the sheet boundary, as if it was a rigid wall. The same configuration as that observed in closed containers, i.e., rolls normal to boundaries is then expected. This is actually confirmed by direct observations, as shown below.

\section{EXPERIMENT}

The purpose of the following series of experiments consists in comparing, at low Prandtl number and for moderate aspect ratios, the behavior of convective structures in closed and open containers. Each of the following structures, straight rolls, foci, focus pairs, and textures, have thus been studied in both kinds of containers. For the sake of a meaningful comparison, closed and open containers have been made within the same experimental setup and, for each structure, with the same convective domain geometry.

\section{A. Experimental setup}

The setup has already been described in detail elsewhere $[21,17]$. It is designed so as to achieve and observe convection in argon gas at room temperature and at a Prandtl number of 0.71 .

The top and bottom horizontal plates are made of sapphire and copper, respectively. The top plate is thermally regulated by water circulation and the bottom plate by an electrical heater. The cell is made of Plexiglass and the sheet is made of cardboard. Compared to argon gas $\left(\lambda=1.87 \times 10^{-4}\right.$ $\mathrm{W} \mathrm{cm}{ }^{-1} \mathrm{~K}^{-1}$ at 30 bars and $300 \mathrm{~K}$ ), the thermal conductivity of materials are respectively $2 \times 10^{4}$ (copper), $2 \times 10^{3}$ (sapphire), and 10 (Plexiglass and cardboard) times larger.

Pattern visualization is achieved by the shadowgraph method. Owing to the low density of argon gas at room temperature, increasing the temperature gradients and the optical properties of the medium are necessary for enhancing the contrast of the images. This is obtained by raising the pressure to 30 bars, following a previously described method $[21,17]$.

The cell depth is $1.6 \mathrm{~mm}$ and the critical temperature difference is $3.5^{\circ} \mathrm{C}$. Its uniformity is ensured by three cali- 
brated spacers within an accuracy of $10^{-2} \mathrm{~mm}$. Measurements of pressure reveal its stability at better than $1 \%$. The temperature difference between the top and bottom plates is measured by a series of thermocouples. It is electronically regulated to within $10^{-2}{ }^{\circ} \mathrm{C}$.

Images of the convective layer are made on a chargedcoupled device camera by an afocal doublet of telescopequality lens. The contrast of the images is adjusted by varying the camera position and has been enhanced by image processing.

Except in the study of straight rolls (Sec. III B), the geometries of both the mean flow boundaries and the roll flow boundaries have been taken to be circular. The former, which corresponds to the cell boundary, displays an aspect ratio $R^{\prime}$ (the ratio of its radius to the cell depth $d$ ) of $R^{\prime}=25$. The roll flow boundary is determined by the sheet boundary. Except, in a validation experiment (Sec. II D), its aspect ratio $R$ has been fixed to $R=R^{\prime} / 2=12.5$.

According to the thermal diffusivity $\kappa$ of argon $(\kappa=0.69$ $\mathrm{cm}^{2} \mathrm{~s}^{-1}$ at 30 bars and $300 \mathrm{~K}$ ), the vertical and horizontal thermal diffusion times in the convective domains are $\tau_{v}$ $=d^{2} / \kappa=3.7 \mathrm{~s}$ and $\tau_{h}=R^{2} d^{2} / \kappa \approx 10 \mathrm{~min}$. Since the present experiments aim at studying intrinsic mechanisms of pattern dynamics, only asymptotic states observed beyond transient decays have been considered. Following theoretical analysis [8] and observations [13], this has required waiting times of at least $R^{2} \tau_{h} \approx 25 \mathrm{~h}$, unless limit cycles or stationary states were reached. No hysteresis has been noticed on any of the structures studied.

\section{B. Straight rolls}

Outside defect cores, straight rolls may be considered as a local approximation of textures as far as roll curvature is neglected. From this point of view, they stand as the most natural candidate for modeling textures [4]. However, at low Prandtl number, closed containers have revealed a large difference between the onset of time dependence of straight rolls $(\varepsilon \approx 0.5)$ and that of textures $(\varepsilon \approx 0.1)$. This is sufficent to conclude that straight rolls fail to capture the mechanisms of texture time dependence $[20,21,29,30]$. Although they are disqualified for modeling textures, their behavior in open containers is nonetheless interesting in understanding the sensitivity of patterns to mean flow boundary conditions. We thus report it below.

The open container is made with a cardboard sheet involving a rectangular hole so as to fit the geometry of straight rolls. It delimits a convective domain of dimensions $25 \times 19$ in cell depth units. At the small sides of the rectangle, the sheet is put in close contact with the bottom plate so as to stabilize tangential rolls. At the large sides of the rectangle, the sheet is put at some height above the bottom plate so as to induce normal rolls. By this way, all the roll boundary conditions are compatible with straight rolls parallel to the small side of the rectangle. As expected, they give rise to a straight roll structure close to the convective threshold.

The closed container consists of a rectangular Plexiglass cell filling the entire conductive domain and is in close contact with the top and bottom plates. Rolls normal to all boundaries should then be induced. This tendency is however inhibited by placing, along the smallest sides of the rectangle, two thin cardboard strips in close contact with the bottom plate. Then straight rolls parallel to the smallest sides of the container are actually induced close to the convective threshold.

In both open and closed containers, straight rolls show the same route to time dependence. It is displayed in Fig. 2 in the case of open containers. The wave number is selected [Figs. 2(a) and 2(c)] but, as observed in a number of closed containers $[24,17,29]$, its value changes by defect nucleation each time the skewed-varicose instability is encountered [Figs. 2(b) and 2(d)]; asymptotic states are then stationary until an oscillatory motion of rolls induced by the oscillatory instability [4] occurs at high values of $\varepsilon$. The only noticeable difference regarding the kind of container is thus at most quantitative, but, as shown in Fig. 2(e), small enough to conclude: the route to time dependence of straight rolls is independent of the mean flow boundary condition.

\section{Foci}

The open container is made with a cardboard sheet involving a circular hole so as to fit the geometry of foci. In order to generate a roll tangent to the boundary [Fig. 1(b)], the sheet is placed in close contact with the bottom plate. The aspect ratios are $R=12.5$ for the convective domain and $R^{\prime}=2 R$ for the conductive one.

The closed container is achieved by taking a circular Plexiglass cell filling the entire conductive domain $R<r<R^{\prime}$. Then a thin cardboard strip is placed all along its inner boundary in close contact with the bottom plate so as to induce a circular roll there.

Experimental observations show a similar route to time dependence in both closed and open containers [18] (Figs. 3 and 4): The focus singularity first shifts as $\varepsilon$ increases, the pattern being still stationary [Figs. 3(a) and 4(a)]. The amplitude of this off-centering is similar in both kinds of containers, a bit larger in closed containers, however (Fig. 5).

The first dynamical event appears at $\varepsilon=0.20$ in both closed and open containers and consists in defect nucleation by roll pinching at $r \approx 3 R / 4$ [Figs. 3(c) and 4(b)]. In both configurations, two dislocations are generated and climb on a circular roll, one on the left of the off-centering direction, the other on the right. They thus rotate in opposite directions but eventually glide to the focus where they disappear, as illustrated in Figs. 3(c)-3(f) for the open container and Figs. 4(b)-4(f) for the closed container. At this time, a roll pair has been lost. However, the focus singularity generates it back and allows the same scenario to resume. One thus obtains a limit cycle, as already observed in containers with similar aspect ratios $[31,18,17]$. An important difference between containers is in order however: whereas foci show permanent oscillations in closed containers as soon as $\varepsilon=0.20$, they are able to restabilize in open containers in between $0.20 \leqslant \varepsilon \leqslant 0.25$ [Fig. 3(b)].

This periodic dynamics contrasts with that reported in smaller [15] or larger aspect ratios [19,32] where no limit cycles involving defect nucleation have been observed. In particular, in the latter case, foci emit phase traveling waves but fail in reaching a stable state as soon as defects are nucleated: their center then moves towards the sidewalls where it disappears, leaving a textured structure. 


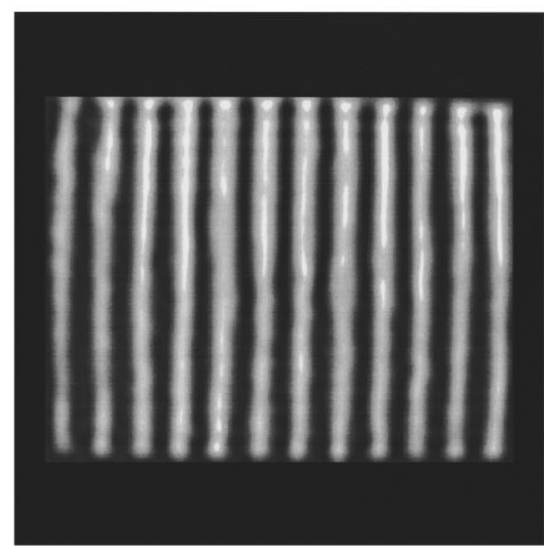

(a)

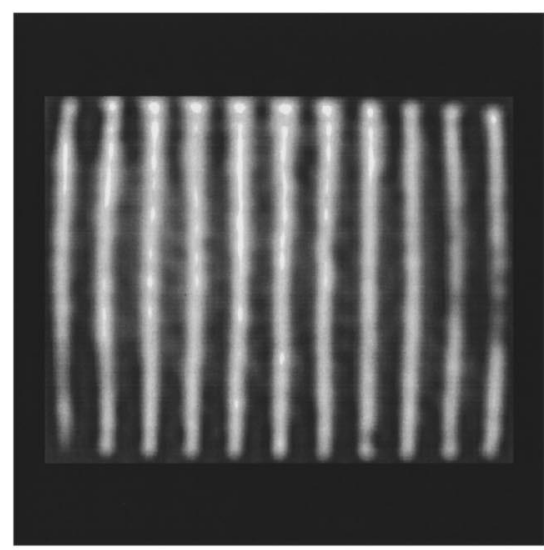

(c)

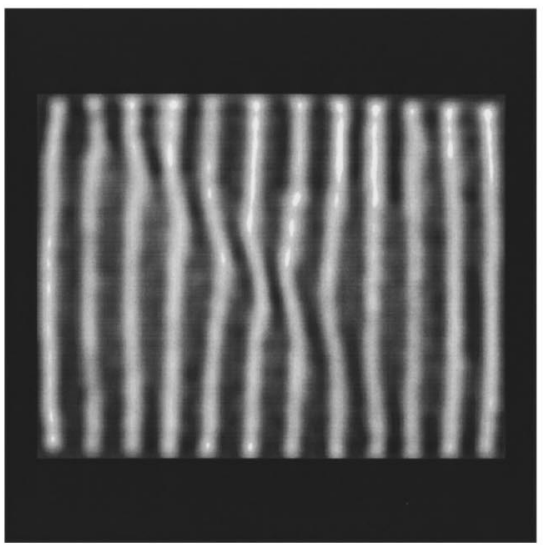

(b)

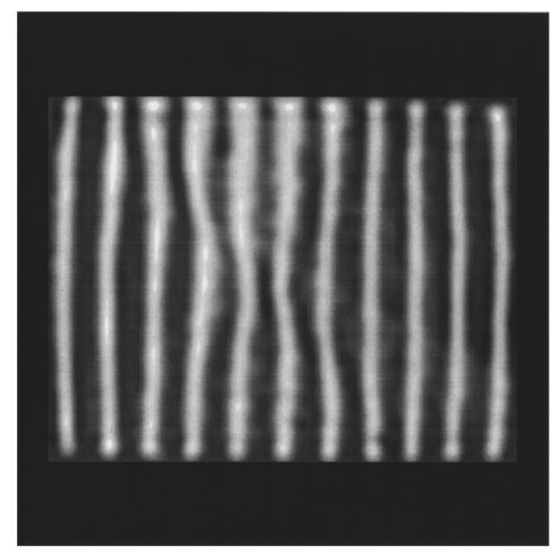

(d)

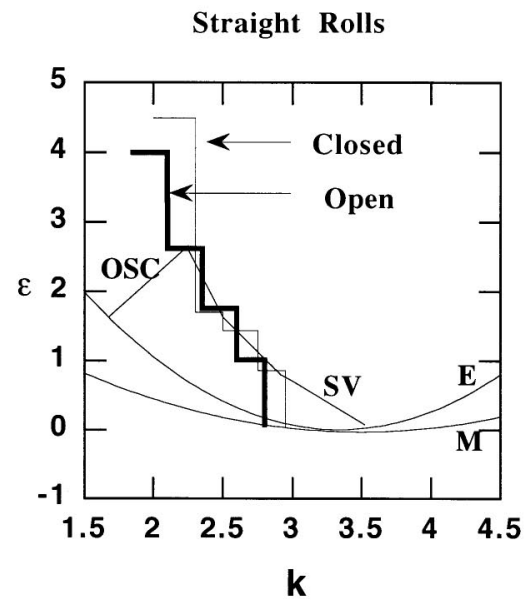

(e)

FIG. 2. Instability of straight rolls in an open container: $\Gamma_{x} \Gamma_{y}=25 \times 19$. (a) Stationary state: $\varepsilon<1.01$, (b) skewed-varicose instability: $\varepsilon=1.01$, (c) stationary state: $1.01<\varepsilon<1.75$, (d) skewed-varicose instability: $\varepsilon=1.75$, (e) stability diagrams in closed and open containers: $\varepsilon$ is the reduced Rayleigh number and $k$ the wave number of straight rolls; the symbols M, E, SV, and OSC refer to the marginal, the Eckhaus, the skewed-varicose, and the oscillatory stability curves.

A more accurate observation of the limit cycles reveals that, in both containers, the motion of the two dislocations is not synchronous. Near the onset of the dynamics, the dislocation which climbs clockwise moves quicker than the other [Figs. 3(d) and 4(d)], and, in closed containers, even disappears sooner at the focus [Fig. 4(e)]: the period is then about $20 \mathrm{~min}$, i.e., $2 \tau_{h}$. As $\varepsilon$ increases, dislocations are nucleated closer to the sidewalls $(r \approx R)$ and are better synchronized: the dynamics becomes more and more symmetric and the period decreases to about $3 \mathrm{~min}$, i.e., $0.3 \tau_{h}$, at $\varepsilon=0.36$.

At this value of $\varepsilon$, the limit cycles show a period doubling in both kinds of containers: dislocations are still not synchronous but the quickest dislocation changes at each cycle, one time that climbing clockwise, the other time that climbing counterclockwise. Labeling the clockwise direction "+" and the counterclockwise direction "-" the dynamics 


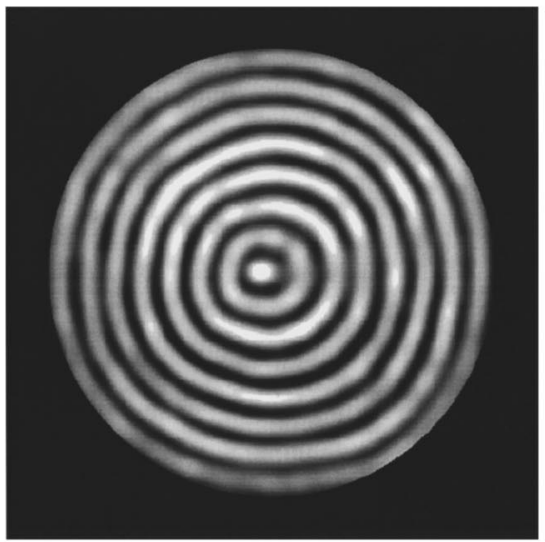

(a)

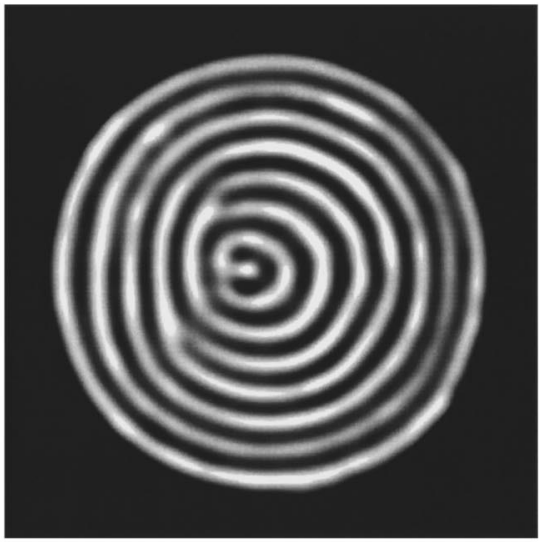

(d)

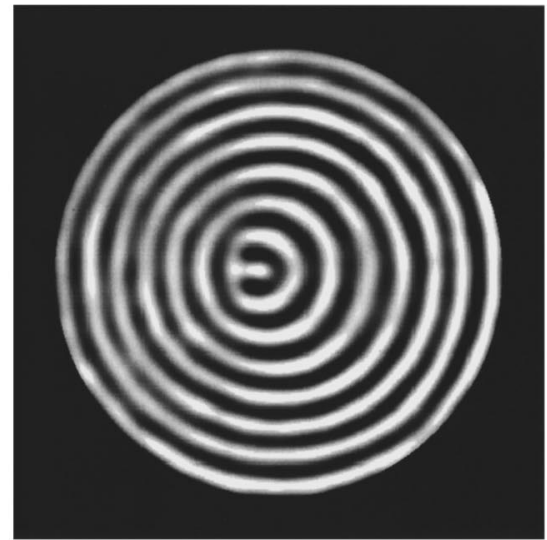

(b)

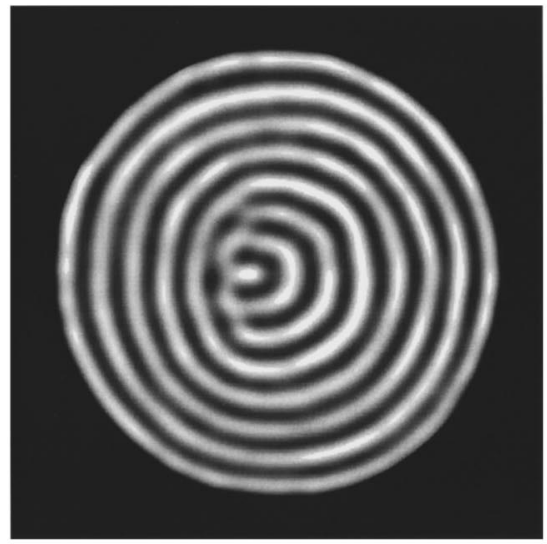

(e)

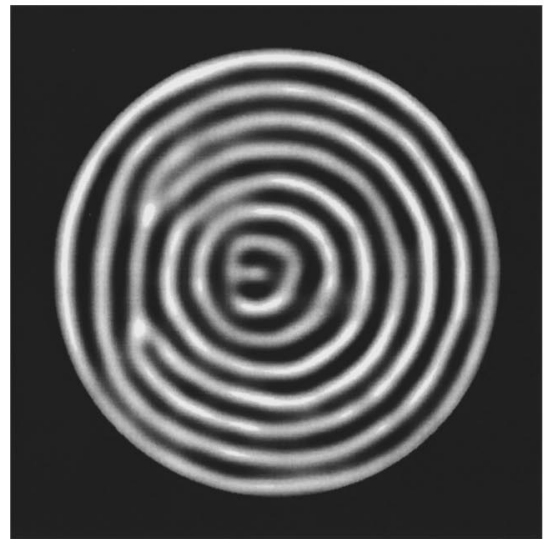

(c)

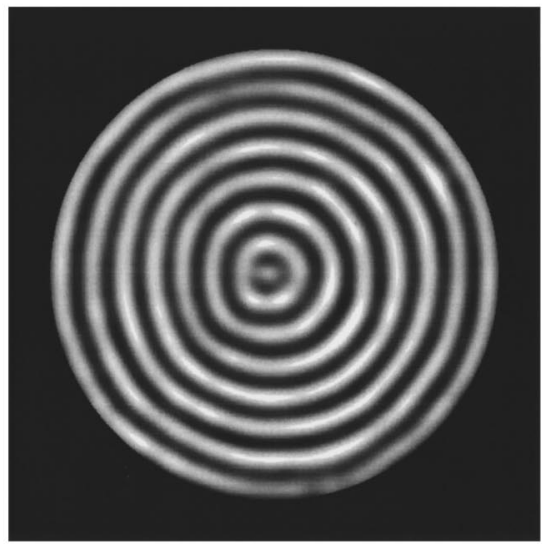

(f)

FIG. 3. Instability of foci in an open container: $R=12.5, R^{\prime}=25$. (a) Stable focus $\varepsilon<0.20$. An off-centering of the focus singularity is noticeable, (b) Stationary state: $0.20<\varepsilon<0.25$, (c)-(f) Time-dependent focus: $0.25<\varepsilon$. Notice the small asymmetry of defect climbing in (d).

may then be symbolized by the series of directions displayed by the quickest dislocation: $(+,-,+,-, \ldots)$.

A period-four regime is then observed at $\varepsilon=0.42$. It is induced by the nucleation of another dislocation pair before the previous pair has disappeared. Although both pairs are simultaneously present for a while, their dislocations never collide, the slowest dislocation of the oldest pair reaching the focus center before the quickest dislocation of the youngest one. Their coupling, however, modifies the dynamical sequence, the quickest dislocation showing the same direction during two cycles before switching to the other direction. This generates the following series of quickest dislocations: $(+,+,-,-,+,+, \ldots)$. The states referring to the simultaneous presence of consecutive pairs of dislocations may be identified by quoting the couples of their quickest dislocations. They then correspond to the series $[(+,+),(+,-),(-,-),(-,+),(+,+), \ldots]$, and thus to a periodfour regime.

We emphasize that the change from the period-two regime to the period-four regime does not correspond to a modulation of the former regime $(+,-,+,-, \ldots)$ but to a modification of its switching period from one dynamical state $(+)$ to its symmetric $(-):(+,+,-,-,+,+, \ldots)$. This bifurcation should therefore not be confused with a usual period doubling. As for the similar bifurcations of focus pair dynamics, its origin may trace back to symmetry breaking of the mean flow configuration [13].
Regarding the transition to time dependence, the only difference with respect to the kind of container is thus a restabilization of foci in open containers until $\varepsilon=0.25$. Since this delay is quite short, their route to time dependence may be considered as nearly independent of the mean flow boundary condition.

\section{Focus pairs}

The open and closed containers are the same as those used for foci except that the sheet is placed at a quarter of the cell height in order to allow rolls normal to boundaries [Fig. $1(\mathrm{c})]$. The aspect ratio of the cell and of the convective domain are still $R^{\prime}=2 R$ and $R=12.5$. The main geometry of focus pairs is shown in Fig. 6(b) in the case of open containers.

Experiments reveal qualitative similarities but large quantitative differences between the routes to time dependence of focus pairs according to the kind of container.

\section{Closed container}

The observed route to time-dependence is the same as that reported in the literature $[21,17,19]$. Focus pairs display wave-number gradients and, especially, a roll compression on the line joining foci. The largest compression is reached at the pattern center, on the central roll separating foci. Quite near the onset of convection, at $\varepsilon=0.08$, this roll becomes 


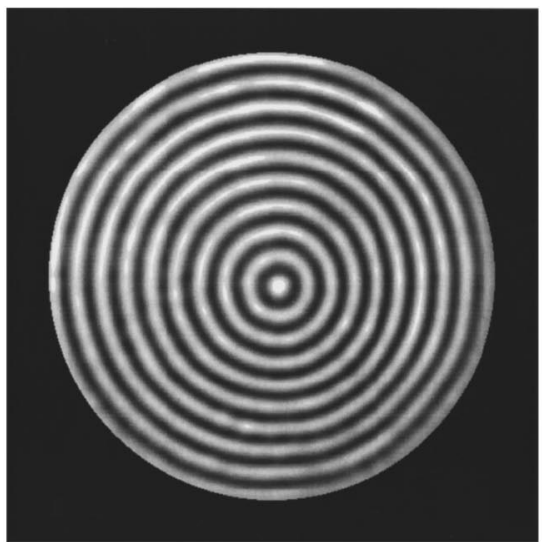

(a)

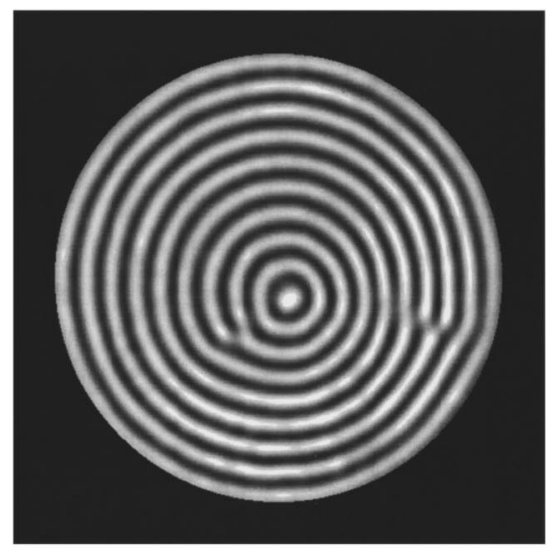

(d)

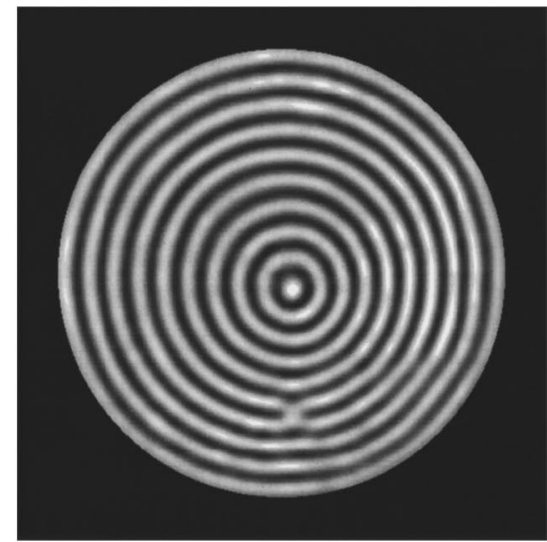

(b)

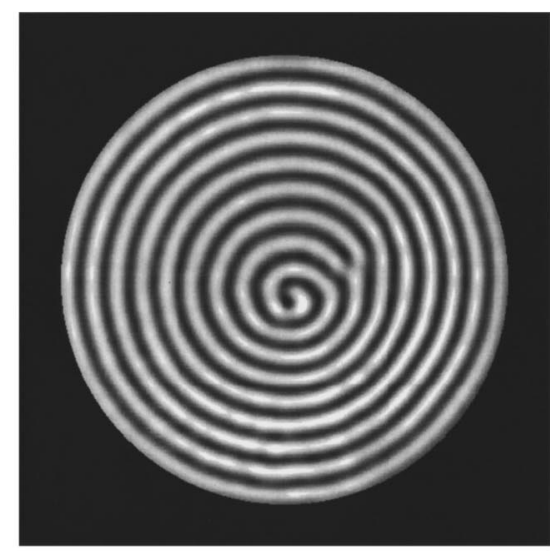

(e)

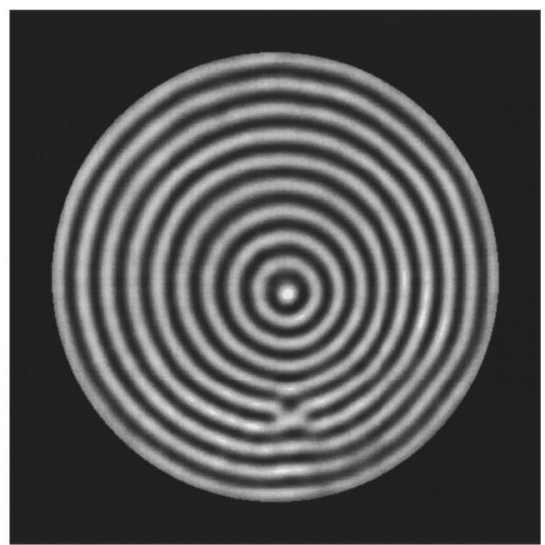

(c)

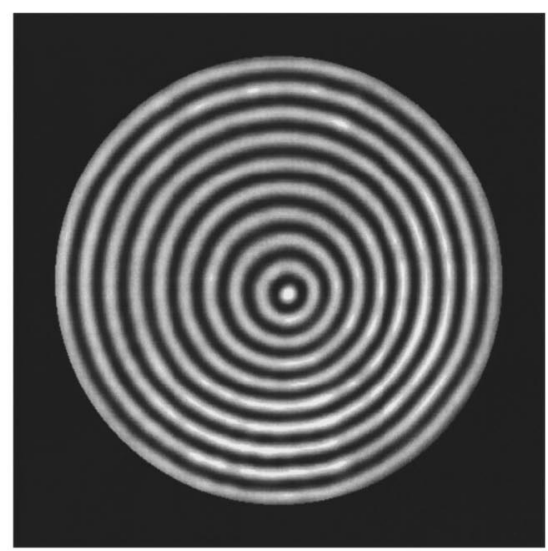

(f)

FIG. 4. Instability of foci in a closed container: $R=12.5, R^{\prime}=R$. (a) Stable focus $\varepsilon<0.20$. An off-centering of the focus singularity is noticeable, (b) Defect nucleation: $\varepsilon=0.20$, (c) $-(\mathrm{e})$ Defect evolution by climbing and gliding, (f) Elimination of defects at the focus singularity. Notice the large asymmetry of defect climbing in (d).

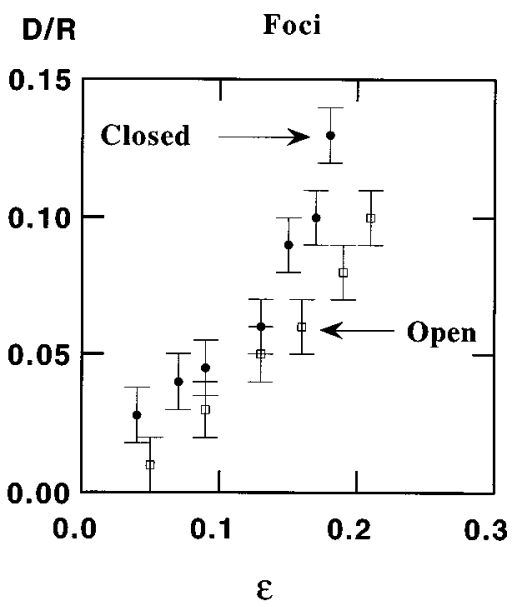

FIG. 5. Measurement of the reduced off-centering $D / R$ of stable foci before time dependence. $D$ represents the distance between the center of the smallest roll and the geometrical center, $R$ the radius of the pattern, and $\varepsilon$ the reduced Rayleigh number. The evolution is continuous from the onset of convection, in contradiction with the concept of spontaneous instability beyond some distance from the onset of convection. Black circles refer to closed containers $(R$ $=12.5)$ and open squares to open containers $\left(R=12.5, R^{\prime}=2 R\right)$. unstable and shrinks, yielding the nucleation of a dislocation pair. These defects climb and glide to the sidewalls where they disappear, leading back to a defectless focus pair. As this structure involves less rolls than the original focus pair, all of them are less compressed and actually stable. This does not imply steadiness, however. Instead, this focus pair displays a slow evolution at large scale increasing its compression until a new dislocation nucleation occurs. A new cycle then repeats generating a spatiotemporal periodic dynamics.

Farther from onset, till $\varepsilon=0.45$, a detailed study of the dynamics [13], not undergone here, reveals bifurcations of the limit cycle explained by successive symmetry breakings of the mean flow field. A stationary state is then displayed in between $0.45<\varepsilon<0.66$ before an aperiodic persistent dynamics occurs for $0.66<\varepsilon$.

Quantitative evolutions of pattern distortion on the route to time dependence are provided by local wave-number measurements. Figure 7 displays those performed at the most and least compressed points of steady focus pairs: the pattern center and the end of the central roll, respectively. The wave number at the pattern center grows until the vicinity of the stability boundary of infinite straight rolls is reached. Then a local instability is triggered there together with the bifurcation to time dependence. 


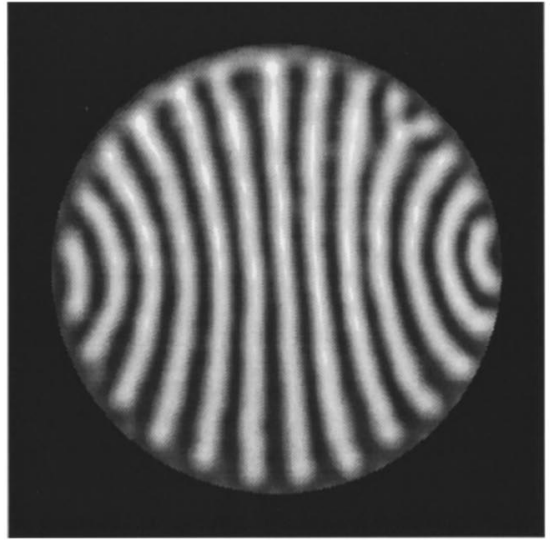

a

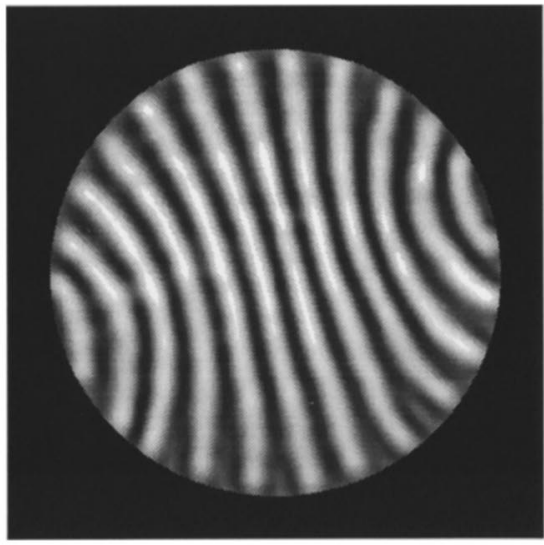

C

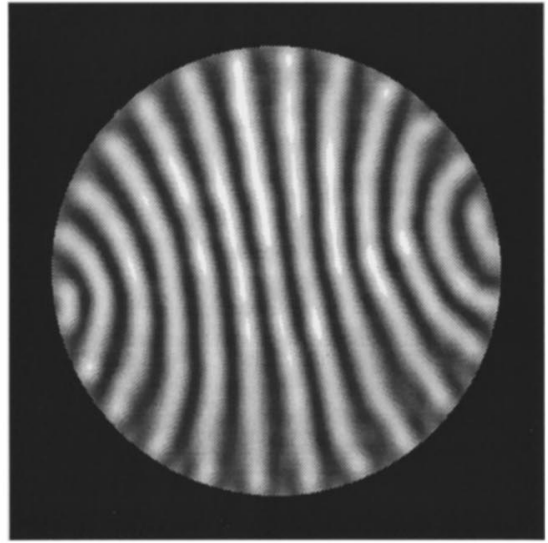

b

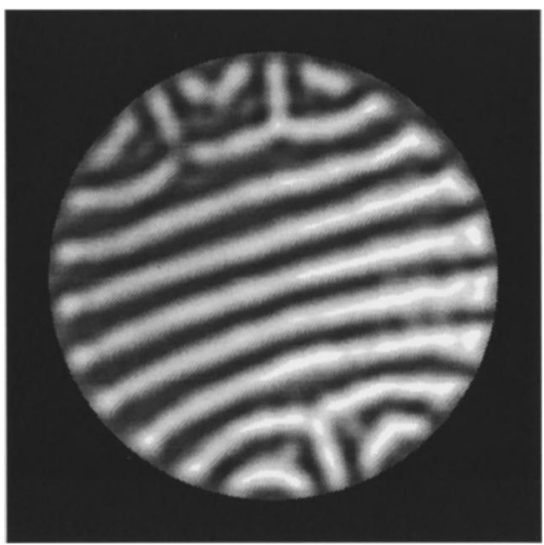

d

FIG. 6. Stationary patterns in open containers: focus pairs in (a) - (c) and texture in (d). $R=12.5, R^{\prime}=25$. (a) $\varepsilon=0.03$. A dislocation has been nucleated by a localized Eckhaus instability near the boundary of the upper-right quarter, (b) $\varepsilon=0.40$. Focus pair: rolls are still perpendicular to the boundaries, (c) $0.56<\varepsilon<0.60$. Focus pair: one roll pair has been lost by localized instability and defect elimination. (d) $0.74<\varepsilon<1.2$. Stationary texture.

\section{Open container}

We describe the route to time dependence in open containers for increasing $\varepsilon$ : Close to onset, $\varepsilon<0.1$, the roll curvature is too weak for providing rolls normal to boundaries. At $\varepsilon \approx 0.1$, a dislocation spontaneously occurs by roll pinching at the boundary of the central roll [Fig. 6(a)]. It remains at this place until it reaches some slightly higher value of $\varepsilon$ and then disappears by gliding to a focus. A steady focus pair is then displayed up to a surprisingly large value of $\varepsilon$ : $\varepsilon=0.56$ [Fig. 6(b)].

At $\varepsilon=0.56$, it undergoes a defect nucleation at the pattern center in a way similar to that displayed in a closed container (Fig. 8). Especially, after elimination of defects at focus centers, the new focus pair hereto involves stable rolls. However, in contrast to the behavior observed in closed containers, it displays no evolution at a large scale. It has thus reached a small-scale equilibrium (any roll is stable), as well as a large-scale equilibrium (the roll patches are steady) [Fig. 6(c)] and is thus stationary. It contains one roll pair less than the previous focus pair, however.

This second focus pair remains stationary until $\varepsilon=0.74$. It then undergoes defect nucleation and restabilizes in a stationary pattern again. However, in contrast with the previous case, its geometry is more complex than a focus pair and displays, as shown in Fig. 6(d), several foci joined by grain boundaries. It remains stationary until $\varepsilon=1.2$.

Above $\varepsilon=1.2$, no stationary states have been observed, despite very large waiting times of order $R^{2} \tau_{H}$ (Figs. 9 and 10). The corresponding time-dependent states will be described in Sec. III E.

Local wave numbers of steady focus pairs have been measured at three locations: the pattern center $k_{\mathrm{pc}}$, the foci $k_{f}$, and the boundary of the central roll $k_{b}$. The corresponding values are displayed till $\varepsilon=0.74$ on Fig. 7 .

The wave number $k_{b}$ is quite close to the Eckhaus instability. This is consistent with the nucleation of a dislocation 

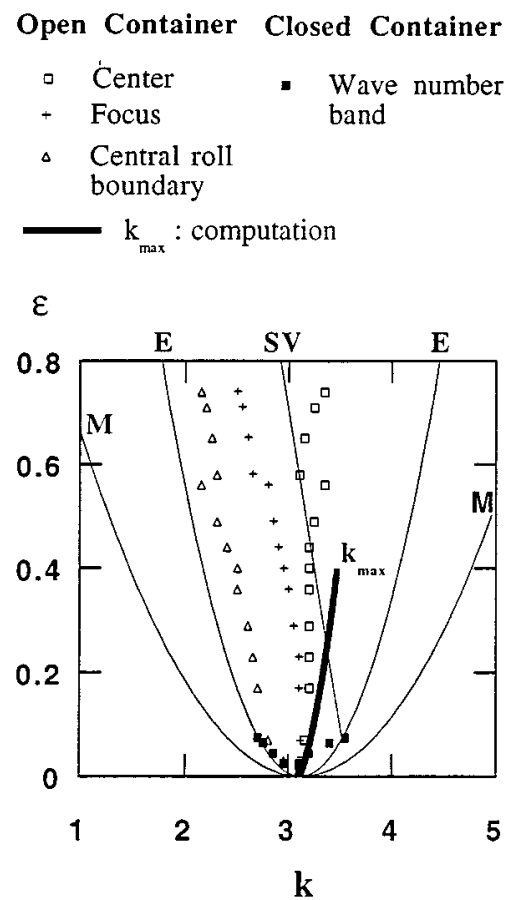

FIG. 7. Stability diagram of infinite straight rolls at $\mathrm{Pr}=0.7$, displaying the marginal (M), the Eckhaus (E), and the skewedvaricose (SV) stability curves. $\varepsilon$ and $k$ denote reduced Rayleigh numbers and wave numbers. We have plotted the local wave numbers measured on focus pairs in closed and open containers. Black squares correspond to the band of wave numbers in a closed container. Open squares, crosses, and triangles correspond to the wave numbers in an open container, at the pattern center $k_{\mathrm{pc}}$, the focus $k_{f}$, and the boundary of the central roll $k_{b}$, respectively. The bold line shows the maximal wave number $k_{m}$ displayed by the solution of the Cross-Newell equations in open containers, the viscous stress of the annular sheet being taken into account. It is computed from the relations (20) and (21), and (D1)-(D3) of Appendix D for $p=0$, $a=\frac{2}{3}, \operatorname{Pr}=0.7, \rho=2$, and $\delta=\frac{1}{4}$. As it should be reached at the pattern center, it should correspond to $k_{\mathrm{pc}}$.

at the boundary of the central roll for $\varepsilon \approx 0.1$ [Fig. 6(a)]. On the other hand, the wave numbers $k_{\mathrm{pc}}$ and $k_{f}$ show similar values. Since their difference results from the phase advection by the mean flow on the axis joining the pattern center to a focus, this indicates that mean flows display an amplitude weaker than in a closed container and/or that they change direction on this axis.

At the transition between focus pairs $(\varepsilon=0.56)$, the wave number at the pattern center lies slightly inside the instability domain of infinite straight rolls with respect to the skewedvaricose instability (Fig. 7). Any other local wave number is stable however. This agrees with the observation of a single local instability at the pattern center displaying a roll modulation analogous to a skewed-varicose distortion (Fig. 8). The slight difference between the marginal stabilities of straight rolls and focus pairs is not surprising owing to the finite size of the container and the spatial inhomogeneity of the structure.

Finally, as may be noticed in Fig. 7, the new focus pair displays a reduced wave-number band that fits entirely into the stable domain of straight rolls. In agreement with our observations, its local stability is then restored.

\section{Comparison between closed and open containers}

In both kinds of containers, the route to time dependence of focus pairs shows similar qualitative features, especially roll compression and roll pinching. This suggests that the mechanism for time dependence is presumably the same in both cases. However, quantitative comparison of wave numbers points out that, although the wave-number band quickly explodes in closed containers, it remains nearly constant in open ones (Fig. 7). The main destabilizing factor of focus pairs, roll compression, has thus been largely weakened by opening the container. This results in a large delay of the onset of persistent time dependence $\varepsilon_{0}$ as big as an order of magnitude: $\varepsilon_{0}=0.74$ in open containers instead of 0.08 in closed containers.

\section{E. Textures}

The closed and open containers are designed so as to allow rolls normal to boundaries. They are thus the same as those used for focus pairs. In either closed or open containers, textures [Figs. 6(d), 9, and 10] show the following important properties: except for a few marginal cases [13], the dynamics beyond transient decay is independent of the kind of texture chosen as initial condition, of the way the onset of convection is crossed (slowly or suddenly), and more generally, on the history. This legitimizes the concept of a common route to time dependence for textures. Moreover, in each kind of container, textures and focus pairs show the same route to time dependence: the same asymptotic states, the same onsets of time dependence, and the same events triggering dynamics by local instabilities and defect nucleation. Especially, we emphasize that textures show the same spectacular inhibition of time dependence in open containers than focus pairs, whatever their initial condition.

We now focus attention to texture behaviors in open containers. As in closed containers [13], their relaxation time to asymptotic states is quite long, usually of the order of several $R \tau_{h}$, except at bifurcation points where it varies in a large range: it is of the order of a few $\tau_{h}$ only at the transition between focus pairs $(\varepsilon=0.56)$ but lasts as long as $R \tau_{h}$ at the transition to complex stationary structures $(\varepsilon=0.74)$. This suggests that stationary attractors are weakly attracting in phase space and are few in number, so that a long wandering is necessary to reach them.

Above $\varepsilon=1.2$, a persistent time dependence of textures is displayed in open containers (Figs. 9 and 10). Two different types of dynamics may be distinguished, depending on the scale of the destabilized spatial modes. From $\varepsilon=1.2$ to $\varepsilon=1.5$, patterns are still in equilibrium at a large scale, but not at a small scale. They then show localized dynamical events involving periodic cross-roll-like instabilities or grain-boundary motions, but no evolution of the large-scale geometry (Fig. 9). Above $\varepsilon=1.5$, pattern equilibrium is destroyed both at large and a small scale: large-scale erratic evolutions occur, together with defect nucleations, smallscale instabilities, and rotating spirals reminiscent of those recently observed in larger aspect ratios [33,34] (Fig. 10).

We finally notice that, in any dynamical regime, transient or turbulent, a phenomenon specific to open containers is 


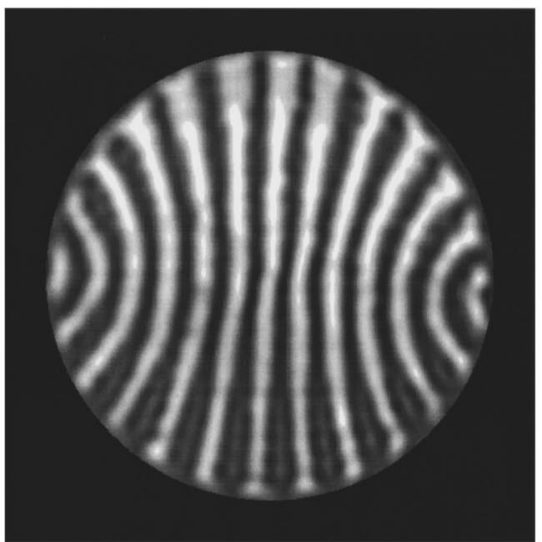

(a)

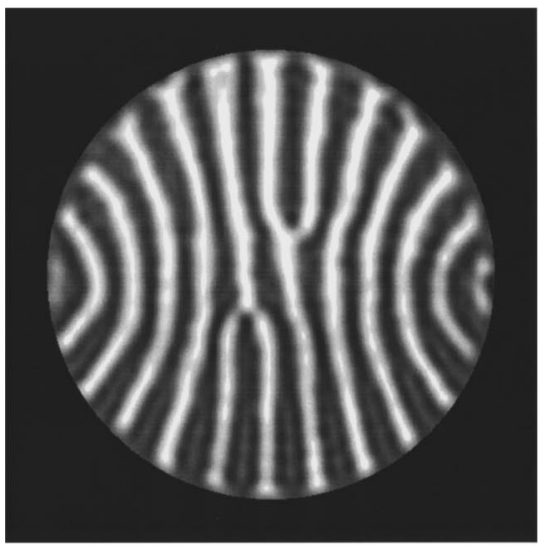

(d)

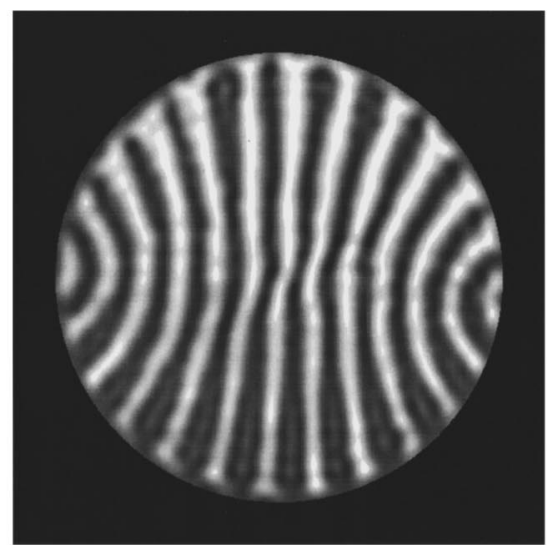

(b)

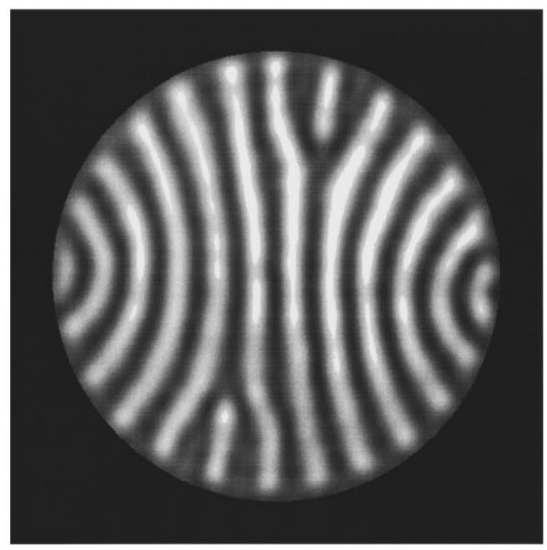

(e)

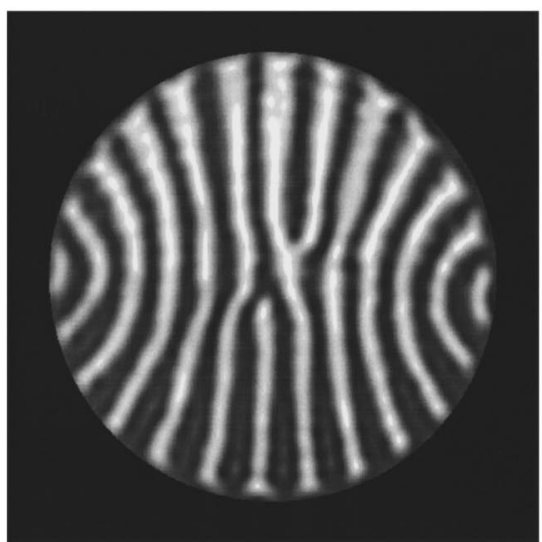

(c)

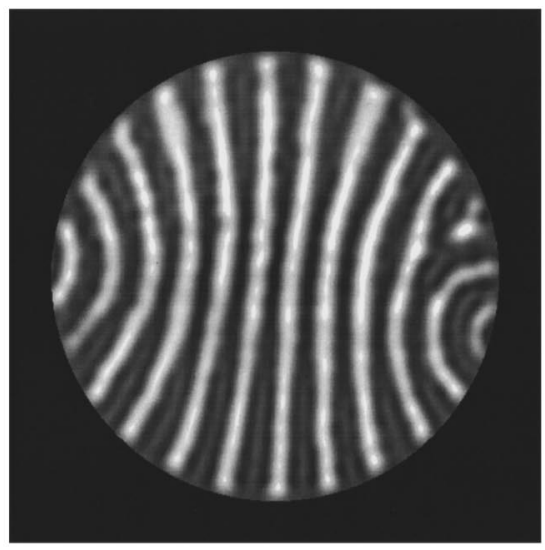

(f)

FIG. 8. Transition between stationary focus pairs in open containers: $\varepsilon=0.56, R=12.5, R^{\prime}=25$. (a) Localized skewed-varicose instability at the pattern center, (b), (c) Defect nucleation, (d)-(f) Defect elimination at the foci.

displayed: focus singularities not only generate new rolls as in closed containers, but also sometimes absorb rolls.

\section{F. Conclusion}

Convective structures display two opposite sensitivities to a change of mean flow boundary conditions: (i) quasiinvariance of spatiotemporal features including the onset of time-dependence, (ii) large modification of spatiotemporal features including a weakening of roll compression and a spectacular delay of the onset of time-dependence.

These experimental evidences reveal two kinds of dynamics corresponding to two classes of structures.

(i) Boundary independent dynamics. This class includes straight rolls and foci. It is not related to the degree of stability of structures since straight rolls involve the most stable structures whereas foci show time dependence much closer to onset of convection at low Prandtl number. However, we notice that each of these model structures display continuous symmetries of the wave-vector field: translational symmetry for straight rolls and rotational symmetry for foci.

(ii) Boundary sensitive dynamics. This class includes focus pairs and textures. We notice that all of them display the same degree of stability: low or high in closed or open containers, respectively, and at low Prandtl number. We also emphasize that none of these structures displays continuous symmetries of the wave-vector field. Only the most regular structures, the focus pairs, involve a discrete symmetry since all substructures delimited by the central roll and the line joining foci are superposable. Focusing attention here on continuous symmetries only, we shall thus consider them as asymmetric.

\section{ANALYSIS}

This section aims at clarifying, by analytical study of model structures, the origin of the two kinds of sensitivity to boundary condition evidenced experimentally. It is based on the assumption according to which the experimental difference between closed and open containers is purely hydrodynamical and only traces back to a separation of the mean flow boundary from the roll flow boundary. This is actually supported by the experimental evidence of unchanged behavior when these boundaries are distinct, but close to one another (see Sec. II D).

A suitable framework for studying the consequences of a change of mean flow boundary conditions is the CrossNewell equations. It will be applied for the two types of structures relevant to each kind of sensitivity: those involving continuous symmetry of the wave-vector field (hereafter called symmetric structures) and those involving none (hereafter called asymmetric structures). The sensitivity of each of them will be derived. This will yield the link between geom- 


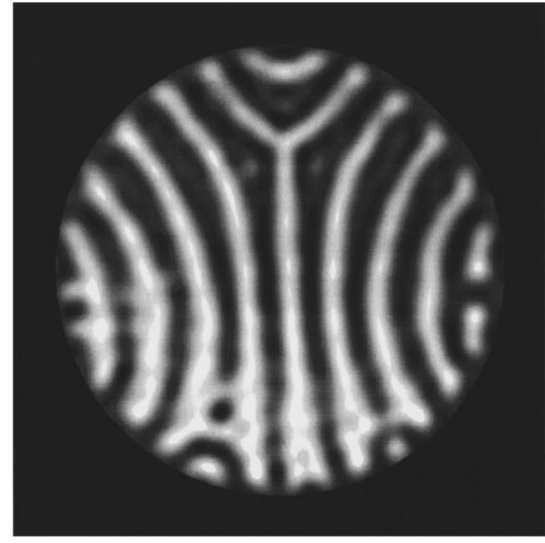

(a)

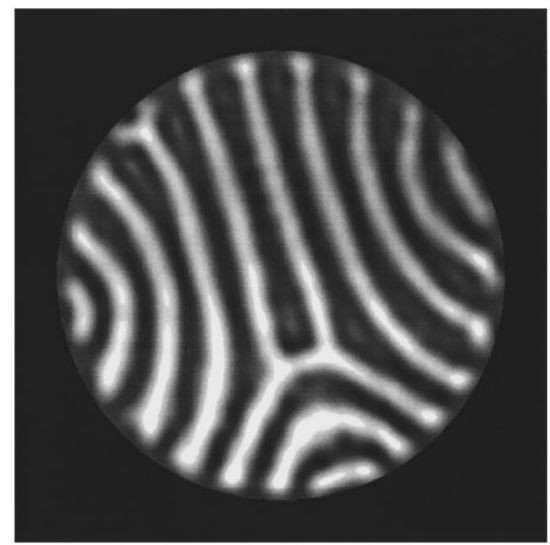

(b)

FIG. 9. Patterns showing local dynamics (only a part of the pattern is unsteady) and large-scale equilibrium (pattern geometry is steady at large-scale) in an open container: $1.2<\varepsilon<1.5, R=12.5$, $R^{\prime}=25$. (a) Localized cross-roll instability at the bottom left of the picture, (b) Grain-boundary motion.

etry and dynamics in this extended convective system.

In the following, the indexes $i, o$, and $I$ will refer to the inner convective zone, the outer conductive zone, and the interface in-between [Fig. 1(a)].

\section{A. The model}

\section{The Cross-Newell equations}

The exact form of the large-scale equations of convection governing the coupled dynamics of the phase field $\varphi$ and the mean flow field $\mathbf{F}$ has been obtained by Newell, Passot, and Souli from the Boussinesq equations [10]. It closely resembles the Cross-Newell (CN) equations [8] previously derived from approximate models of convection, with negligible corrections close to onset of convection $(\varepsilon \subseteq 0.5)$. Since the exact equations are more complex to use than the $\mathrm{CN}$ equations but validate their main features, we prefer to work with the latter in the following:

$$
\begin{aligned}
& \tau\left[\frac{\partial \varphi}{\partial t}+\mathbf{k} \cdot \mathbf{F}\right]+\boldsymbol{\nabla} \cdot(\mathbf{k} B)=0+o\left(\frac{1}{R}\right), \\
& \mathbf{F}=-\gamma \mathbf{k} \boldsymbol{\nabla} \cdot\left(\mathbf{k} A^{2}\right)+\boldsymbol{\nabla}(\Pi)+o\left(\frac{1}{R}\right) .
\end{aligned}
$$

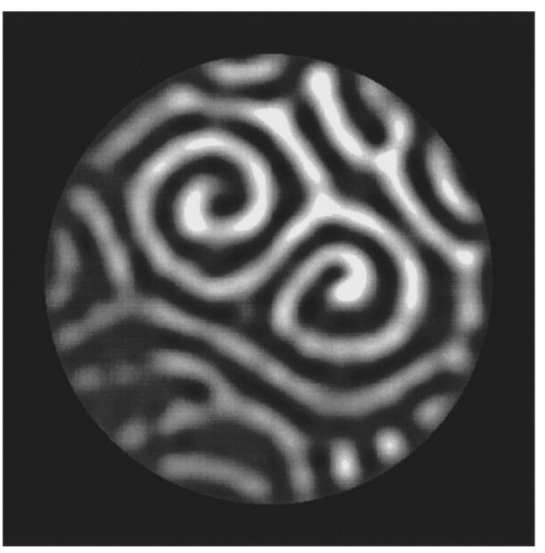

FIG. 10. Phase turbulence in an open container: $1.5<\varepsilon$, $R=12.5$, and $R^{\prime}=25$. Notice the rotating spiral reminiscent of those observed in spiral defect chaos [33,34].

Here $\mathbf{k}=\boldsymbol{\nabla} \varphi$ is the phase gradient, $A$ the roll amplitude, $\Pi$ a pressure field, and $B(k, \operatorname{Ra}, \operatorname{Pr}), \tau(k, \operatorname{Ra}, \operatorname{Pr})$, and $\gamma(\operatorname{Pr})$ suitable scalar functions, $\gamma$ being nearly proportional to $\mathrm{Pr}^{-1}$.

The physics of these equations is recalled in Appendix A. Their validity is restricted to first order in the inverse aspect ratio $1 / R$. Moreover, the mean flow equation (2) neglects the mean flow dynamics and is only valid close to the convective threshold $(\varepsilon \ll 1)$.

\section{Hydrodynamic interface}

At a large scale, the interface between the inner and outer zones appears as a discontinuity of the large-scale vorticity [Fig. 1(a)]. Of course, this is not realistic since vorticity is a divergence-free field that cannot vanish abruptly. In fact, some vorticity sources are also generated there, either by mean Reynolds stresses or by mean flow shear.

The small extension of this interface does not allow us to neglect its vorticity contribution, since the short-scale variations induced in it may yield a large vorticity magnitude. Especially, it is shown in Appendix $\mathrm{C}$ that it actually dominates the net mean vorticity generated in the convective domain.

The different kinds of vorticity sources might be difficult to compute separately. Fortunately, their net contribution will be determined directly by using the continuity of the pressure field across the interface (see Appendix B).

\section{Boundary conditions}

We denote by $\mathbf{n}$ the boundary normals. Foci involve a roll tangential to boundary:

$$
\mathbf{k} \times \mathbf{n}=\mathbf{0} \quad \text { at } r=R .
$$

Focus pairs involve rolls normal to boundary:

$$
\mathbf{k} \cdot \mathbf{n}=\mathbf{0} \quad \text { at } r=R .
$$

Mean flows vanish at the impermeable boundary:

$$
\mathbf{F} \cdot \mathbf{n}=\mathbf{0} \quad \text { at } r=R^{\prime} .
$$




\section{B. Symmetric structures}

\section{Definition and approach}

We call symmetric structures, the structures for which the wave-vector field satisfies a continuous symmetry, either translational or rotational. This selects parallel or radial wave vectors and thus straight rolls [Fig. 2(a)] or foci [Figs. 3(a) and $4(\mathrm{a})]$.

The stability analysis of these structures turns out to solve the linearized $\mathrm{CN}$ equations, together with their boundary conditions, for normal modes of perturbations. Linear stability is then deduced from the resulting dispersion relation. For straight rolls, this procedure is readily achieved with Fourier modes since the partial differential equations are homogeneous $[27,8]$. It is however much more complex to implement in foci since the corresponding equations involve space-dependent terms. Evidence of instability is then obtained from integral considerations and numerical calculations $[9,10]$.

In the following, our goal consists in comparing the linear stability analysis of symmetric structures in closed and open containers without deriving explicitly either of them. We shall first notice that their basic state of instability does not depend on the kind of container. This will lead us to focus attention to the modification brought about on mean flows by the sole change of boundary conditions. Analyzing its consequence on the instability spectra will show the independence of the onsets of instability with respect to mean flow boundary conditions.

\section{Mean flow sources}

Since mean flow sources correspond to mean Reynolds stresses, they derive from roll modulation and thus satisfy the same symmetries as the roll structure. Within the convective domain, they then generate, according to Eq. (2), mean flows normal to roll axis in a straight roll structure and radial mean flows in foci, up to a pressure gradient. In addition, the former flows are invariant by translation along the roll axis and the latter are invariant by rotation around the focus center. Owing to these symmetries, no mean flow vorticity can be generated in both cases in the convective domain.

Within the interface, the roll direction is either normal or parallel to the roll boundary, in either straight rolls and foci and in either kinds of containers (Figs. 2-4). The roll structure therefore satisfies a translational symmetry along the interface and a reflection symmetry with respect to the interface normal. Since its mean Reynolds stresses must satisfy the same symmetries, they can only be a vector field parallel to the boundary normal and independent of the orthogonal direction. No field of this kind can generate vertical vorticity. We emphasize that this statement is valid in stable or unstable regimes, since the boundary rolls stay the same anyway.

The mean flows generated by symmetric structures and by boundary rolls can thus only be potential, incompressible, and free of singularity. However, no flow of this kind can exist in a closed cell. Neither symmetric structures in stable states nor their interface in unstable states can therefore generate mean flow, in any kind of container.

\section{Basic state of instability}

Since, whatever the kind of container, symmetric structures involve no mean flow, their phase field is the same in either case. The difference between their stability analysis therefore traces back to a change not of the basic state of instability but of the mean flows. We shall denote $\delta \mathbf{F}$, this mean flow variation induced by the sole change of mean flow boundary conditions. We address its main features below.

\section{Mean flow perturbation}

As mean flows vanish in symmetric structures, whatever the kind of container, the mean flow modification $\delta \mathbf{F}$ brought about by the change of boundary conditions only results from that induced on mean flow perturbations. It is thus at least of the same order as the phase perturbation $\psi$ and displays, at first order in $\psi$, the same growth rate as $\psi$.

On the other hand, according to (2), the mean flow vorticity generated in the convective domain only follows from roll modulation, independently of the kind of container. This means that the change of mean flow boundary condition brings no additional vorticity in the inner zone by itself and thus that the corresponding mean flow modification $\delta \mathbf{F}_{i}$ can only be a potential flow satisfying mass conservation. Both its potential and its stream function therefore satisfy a laplace equation: $\delta \mathbf{F}_{i}=\boldsymbol{\nabla}(\delta p)=\boldsymbol{\nabla} \times\left(\xi \mathbf{e}_{z}\right)$ with $\Delta(\delta p)=\Delta(\xi)=0$.

\section{Dispersion relation}

At first order in phase perturbation, the only difference brought about by the change of containers comes from the mean flow variation $\delta \mathbf{F}_{i}$ through the advection term $\mathbf{k}_{u} \cdot \delta \mathbf{F}_{i}, \mathbf{k}_{u}$ denoting the wave vector of the unperturbed structure. We determine below its consequence on the instability spectrum.

As the additional mean flow $\delta \mathbf{F}_{i}$ and the phase perturbation $\psi$ have the same growth rate, eliminating one of them from the linear stability analysis does not modify the instability spectrum but provides the opportunity of focusing the analysis on essential modes. Elimination of $\delta \mathbf{F}_{i}$ may be achieved as follows: Taking the curl to the mean flow equation (2) yields an equation linking the mean flow vorticity $\Omega$ to the phase perturbation $\psi$. It is decoupled from $\delta \mathbf{F}_{i}$ since $\delta \mathbf{F}_{i}$ drives no vorticity.

On the other hand, applying a suitable differential operator $P(\cdot)$ to the phase equation (1) yields a dynamical equation for the phase perturbation $\psi$ that only involves the mean flow difference $\delta \mathbf{F}_{i}$ via $P\left(\mathbf{k}_{u} \cdot \delta \mathbf{F}_{i}\right)$. When the basic structure consists of straight rolls, $\mathbf{k}_{u}$ is a constant vector $k_{s} \mathbf{e}_{x}$. Taking $P(\cdot)=\Delta(\cdot)$ then yields $P\left(\mathbf{k}_{u} \cdot \delta \mathbf{F}_{i}\right)=k_{s} \partial \Delta(\delta p) / \partial x=0$. On the other hand, when the basic structure is a focus, $\mathbf{k}_{u}$ is a radial vector $k_{f} \mathbf{e}_{r}$. Taking $P(\cdot)=\Delta\left(r k_{f}^{-1} \cdot\right)$ yields $P\left(\mathbf{k}_{u} \cdot \delta \mathbf{F}_{i}\right)=\partial \Delta(\xi) / \partial \theta=0$. In both cases, $\delta \mathbf{F}_{i}$ disappears from the equation and, finally, from the stability analysis.

According to the above statements, the mean flow difference $\delta \mathbf{F}$ between containers cannot modify the dispersion relation and thus the onset of linear instability; it only changes the shape of the unstable modes by driving an additional phase distortion $\delta \psi$ displaying the same growth rate than the other dynamical modes and yielding no mean flow vorticity. Symmetric structures therefore keep the same onset 
of instability in either closed or open containers.

\section{Asymmetric structures}

\section{Definition and approach}

We call asymmetric structures the structures whose wavevector field satisfies no continuous symmetry, either translational or rotational [Figs. 6(d), 9, and 10]. They thus correspond to any structures different from straight rolls or foci and therefore involve some distortion.

Since asymmetric structures differ from straight rolls, they display wave-vector rotations. However, we emphasize that they also involve wave-number gradients: $\boldsymbol{\nabla}(\mathbf{k} \cdot \mathbf{k}) \neq \mathbf{0}$. Otherwise, since $\mathbf{k}$ is a gradient field, $(\mathbf{k} \cdot \nabla) \mathbf{k}=\boldsymbol{\nabla}(\mathbf{k} \cdot \mathbf{k}) / 2+\mathbf{k}$ $\times \boldsymbol{\nabla} \times \mathbf{k}$ would vanish, except at the singular points where $\mathbf{k}$ is not defined. The field lines of $\mathbf{k}$ would then be similar to the stream lines of a steady flow with no total derivative and would thus correspond to straight lines between singular points. Since an intersection of two field lines of $\mathbf{k}$ is a phase singularity, the only possibilities for keeping their density finite would then be either no intersection or a single one in the whole domain. The former case corresponds to a constant wave-vector field, $\mathbf{k}=k_{s} \mathbf{e}_{x}$, and thus to straight rolls. The latter case corresponds to a radial wave-vector field, $\mathbf{k}=k_{f} \mathbf{e}_{r}$, and thus to foci. Both involve continuous symmetries, in contrast with asymmetric structures.

Owing to these wave-vector gradients, asymmetric structures trigger some mean flow sources which, because of the absence of continuous symmetry, generate some mean flow vorticity. They thus cannot be compensated by a pressure gradient, so that the resulting mean flows are necessarily not zero: $\mathbf{F} \neq \mathbf{0}$. This important feature contrasts with the vanishing of mean flow in symmetric structures and makes all the difference between the two kinds of patterns. Especially, the change of container is now suitable for modifying the mean flows of asymmetric structures and consequently their phase, even in their stable regime. Not only the mean flow perturbations but also the basic state of instability may then now depend on the kind of container. Compared to symmetric structures, this provides an additional opportunity of being sensitive to a change of mean flow boundary conditions.

Another important difference brought about by asymmetry is the following. As a result of phase advection $\mathbf{F} \cdot \mathbf{k} \neq 0$, mean flows, whatever their magnitude, stretch the roll wavelength and thus induce a small but continuous wave-number drift along mean flow streamlines [28,12]. Its consequences are enhanced in large aspect ratio cells since, being integrated over long distances, this drift may result in considerable wave-number shifts. This important effect actually corresponds to the accumulation of a nonlinear phase shift from rolls to rolls and thus to a secular behavior in space, the spatial cycles being provided by rolls and the secularity by the wave number increase. Following it, unstable wave numbers may therefore be reached locally so that local instabilities may be triggered prior to any instability of large-scale fields. This, again, contrasts with symmetric structures where an evolution of geometry could only be generated by largescale instabilities.

A priori, the stability analysis of asymmetric patterns might proceed as in symmetric structures, by seeking the dispersion relation of phase perturbations around some basic

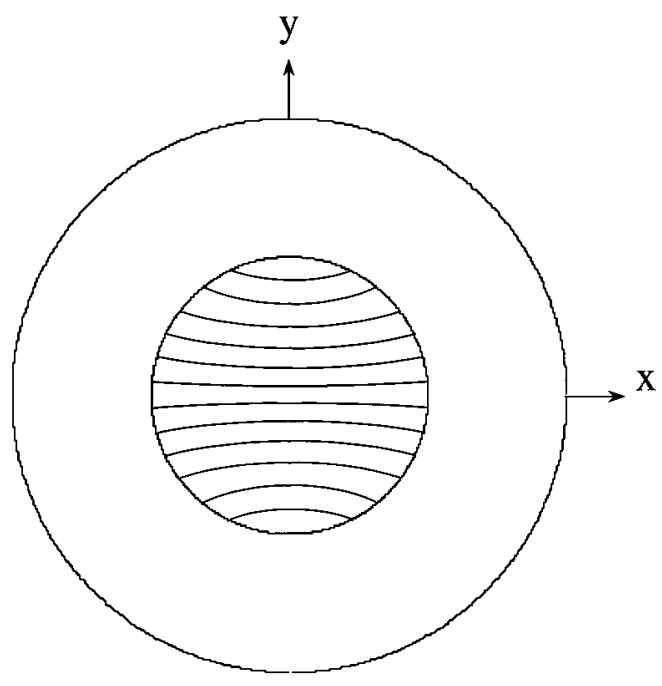

FIG. 11. Sketch of the phase field and the coordinate frame in both closed or open containers.

state. However, owing to the wave-number gradients, the linearized equations would involve space-dependent coefficients that could likely result in a localization of the growth rate $\sigma$ of the perturbations: $\boldsymbol{\nabla}(\sigma) \neq \mathbf{0}$. Especially, in a WKB approximation, a local crossing of the stability boundary of rolls by extremal wave numbers would induce a local positive growth rate and thus a localized instability. Motivated by this statement and by experimental observations, we chose to perform the stability analysis in two steps: first, determination of the basic state of instability and second, investigation of its local stability.

This procedure is implemented below on a model of asymmetric structure: the focus pair. It is similar to that already used in closed containers [11-13] but is supplemented here by an analysis of the conductive zone, of the interface, and of their effects on the convective zone. Owing to the analytical complexity of the $\mathrm{CN}$ equations as far as no continuous symmetry is involved, the basic state of instability is solved by a perturbative method. A relevant polynomial expansion of the phase field is introduced and the resulting mean flows are determined at the same order of expansion. Both fields are then substituted into the phase equation, from which an algebraic system governing the expansion coefficients is obtained. Its solution, compatible with the boundary conditions, provides the identification of the basic state of instability. Its stability at any location is finally investigated by comparison of its local wave numbers with the stability domain of infinite straight rolls, hereafter called the Busse balloon [4].

\section{Phase field}

The central roll line and the line joining foci are denoted $x$ and $y$ axis, respectively (Fig. 11). Following the symmetries of focus pairs with respect to them, the phase field is expanded as

$$
\varphi(x, y)=k_{0}(1+\Delta) y\left[1-a \frac{x^{2}}{R^{2}}+b \frac{y^{2}}{R^{2}}+c \frac{y^{4}}{R^{4}}+d \frac{x^{2} y^{2}}{R^{4}}\right]
$$


where $k_{0}$, the wave number selected by foci, satisfies $B\left(k_{0}, \varepsilon, \operatorname{Pr}\right)=0[8]$.

Here, $a, b, c, d$, and $\Delta$ are expansion parameters. The parameter $a$ drives phase curvature and the remaining ones $b, c, d$, and $\Delta$, phase compression. In agreement with perturbative analysis, they are all considered much smaller than unity. Moreover, following experimental observations showing a weak compression compared to curvature [Fig. 6(b)], we anticipate that $b, c, d$, and $\Delta$ are second order in $a$, as confirmed at the course of the derivation.

\section{Mean flow field}

(a) Mean flow vorticity. Expansion of Eq. (2) yields, according to Eq. (3),

$$
\Omega_{z i}=\omega \frac{r^{2}}{R^{4}} \sin (2 \theta)+o\left(\frac{a^{2}}{R^{2}}\right),
$$

where $\omega=2 \gamma k_{o} A^{2}\left(k_{o}\right) \delta, \quad \delta=a^{2}(1-5 p)-3 d(1+p)$, and $p=\partial \ln \left(A^{2}\right) / \partial \ln k\left(k_{o}\right)$. By symmetry of the underlying pattern, the polar harmonics of the mean vertical vorticity in the interface $\Omega_{z I}$ are even but only the quadrupolar mode is resonant with the other modes of the problem. Disregarding the other harmonics, we thus write

$$
\Omega_{z I}=\frac{\delta(R)}{R} \omega_{I} \sin (2 \theta)+O\left(\frac{\delta(R)}{R^{2}}\right),
$$

where $\delta(R)$ is the delta function and where $\omega_{I}$ will be determined later. Finally, the mean vertical vorticity $\Omega_{z o}$ vanishes in the outer zone:

$$
\Omega_{z o}=0 .
$$

(b) Stream functions; potentials. Owing to the symmetries of the pattern, the stream function $\xi$ of $\mathbf{F}$ is sought as a second polar harmonics: $\xi(r, \theta)=\xi(r) \sin (2 \theta)$. It is obtained by integration of the Poisson equation $\Delta \xi=-\Omega_{z}$ where, according to Eqs. (4) $-(6), \Omega_{z}=\Omega_{z}(r) \sin (2 \theta)$ :

$$
\xi(r)=-r^{2} \int_{0}^{r} \frac{1}{s^{5}} \int_{0}^{s} t^{3} \Omega_{z}(t) d t d s-\frac{\omega}{12}\left[\beta \frac{r^{2}}{R^{2}}+\gamma \frac{R^{2}}{r^{2}}\right] .
$$

We note that $\beta$ and $\gamma$, to be determined later, drive a potential flow. Owing to (4)-(6), $\xi$ may be written in both the inner and outer zones:

$$
\begin{gathered}
r<R: \quad \xi_{i}=-\frac{\omega}{12}\left[\frac{r^{4}}{R^{4}}+\beta \frac{r^{2}}{R^{2}}+\gamma \frac{R^{2}}{r^{2}}\right] \sin (2 \theta), \\
r>R: \quad \xi_{o}=-\frac{\omega}{12}\left[(\beta-\mu+1) \frac{r^{2}}{R^{2}}+(\gamma+\mu) \frac{R^{2}}{r^{2}}\right] \sin (2 \theta)
\end{gathered}
$$

with

$$
\mu=-\left[\frac{1}{2}+3 \frac{\omega_{I}}{\omega}\right]
$$

We shall find it convenient to split the corresponding mean flow fields $\mathbf{F}_{i}=\boldsymbol{\nabla} \times\left(\xi_{i} \mathbf{z}\right)$ and $\mathbf{F}_{o}=\boldsymbol{\nabla} \times\left(\xi_{o} \mathbf{z}\right)$ into a rotational and a potential part, indexed by $r$ and $p$, respectively: $\mathbf{F}_{i}$

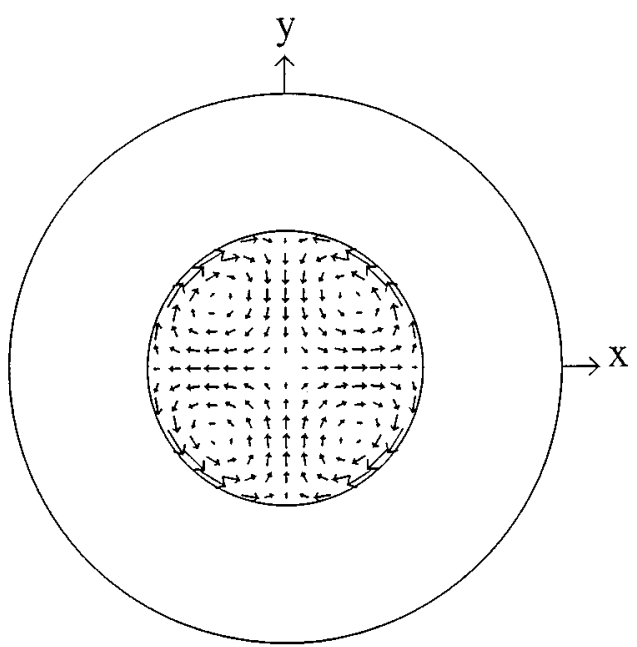

(a)

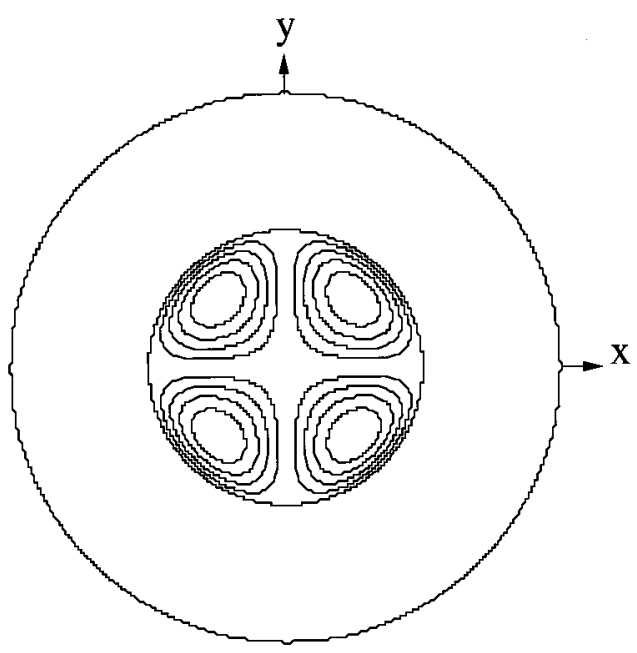

(b)

FIG. 12. Closed container: $\rho=1, \beta=-1$. Sketch of (a) the mean flow field on a square lattice (b) mean flow stream lines for $\lambda=0$, $-0.05,-0.1,-0.15$, and -0.20 . Notice the back flow joining foci and pattern center. This focalization of the mean flow is responsible for a dangerous roll compression at the pattern center.

$=\mathbf{F}_{i p}+\mathbf{F}_{i r}, \mathbf{F}_{o}=\mathbf{F}_{o p}+\mathbf{F}_{o r}$. We make the choice $\mathbf{F}_{o r}=\mathbf{0}$ and $\mathbf{F}_{i r}=\boldsymbol{\nabla} \times\left(\xi_{i r} \mathbf{z}\right)$ where $\xi_{i r}$ is the value of $\xi_{i}$ for $\beta=\gamma=0$. The corresponding potential parts $\mathbf{F}_{i p}$ and $\mathbf{F}_{o p}$ then drive from the following pressure fields:

$$
\begin{gathered}
r<R: \quad \Pi_{i}=-\frac{\omega}{12}\left[\beta \frac{r^{2}}{R^{2}}-\gamma \frac{R^{2}}{r^{2}}\right] \cos (2 \theta), \\
r>R: \quad \Pi_{o}=-\frac{\omega}{12}\left[(\beta-\mu+1) \frac{r^{2}}{R^{2}}-(\gamma+\mu) \frac{R^{2}}{r^{2}}\right] \cos (2 \theta)
\end{gathered}
$$

with $\mathbf{F}_{i p}=\nabla \Pi_{i}$ and $\mathbf{F}_{o p}=\nabla \Pi_{o}$.

(c) Mean flow field. Mean flows satisfy three boundary conditions.

(i) Impenetrability at the cell wall: $\mathbf{F} \cdot \mathbf{n}=0$ at $r=R^{\prime}$. This implies $\xi_{o}\left(R^{\prime}\right)=0$ and thus $\beta=-1+\mu\left(1-\rho^{-4}\right)$ where $\rho=R^{\prime} / R$. 


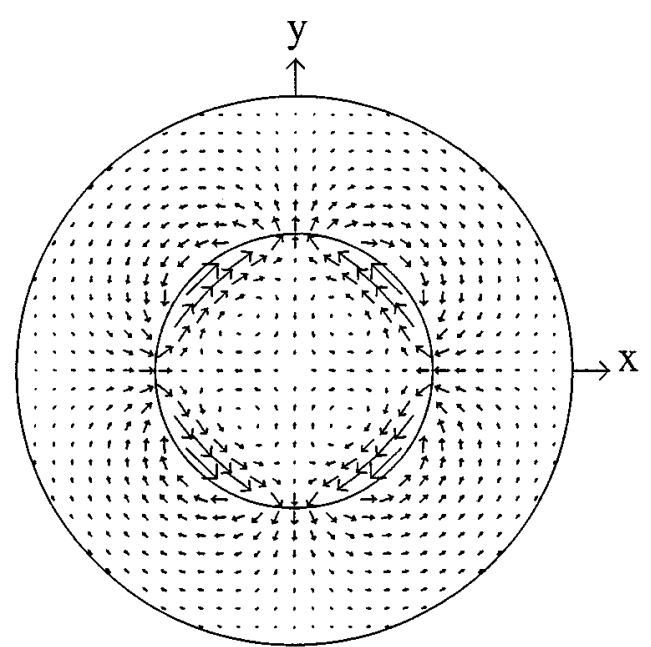

(a)

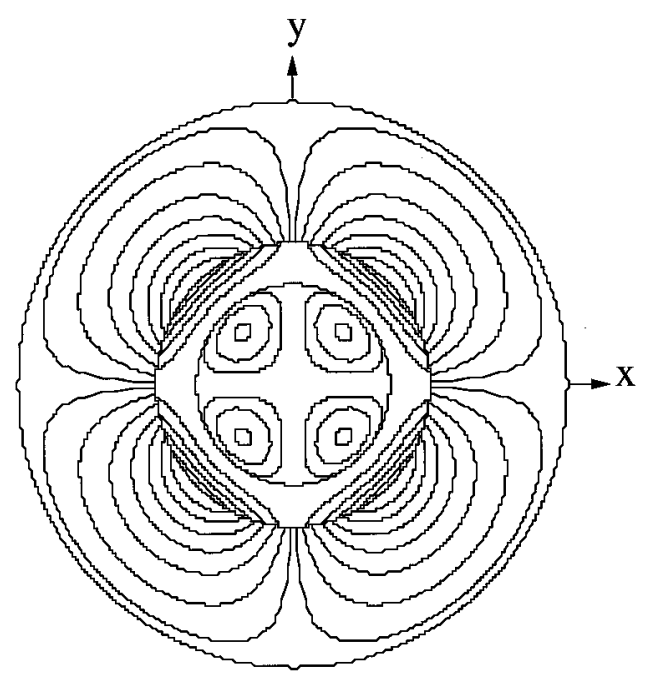

(b)

FIG. 13. Open container: $\rho=2, \beta \approx-\frac{1}{2}$. Sketch of (a) the mean flow field on a square lattice (b) mean flow stream lines $\lambda=0.4,0.3$, $0.2,0.1,0,-0.02,-0.04$, and -0.06 for $r<R$ and $\lambda=n / 32$ with $n=0,0.5,2,4,6,8,10$, and 12 for $r>R$. Notice the mean flow shear at the hydrodynamic interface and the low amplitude of the back flow on the line joining foci and pattern center, compared to that displayed in closed containers. Roll compression at the pattern center is weaker and time dependence is inhibited inside the Busse balloon.

(ii) No singularity at the pattern center. This gives $\gamma=0$.

(iii) Continuity of the pressure fields $\Pi_{i}$ and $\Pi_{o}$ at the interface, as derived in Appendix B. This yields $\mu=\frac{1}{2}$ from Eqs. (11) and (12) and, from Eq. (10), $\omega_{I}=-\omega / 3$. Altogether, these constraints yield

$$
\beta=-\left(1+\rho^{-4}\right) / 2, \quad \gamma=0, \quad \mu=\frac{1}{2} .
$$

We note that the value of $\beta$ changes from -1 to $-\frac{1}{2}$ from closed $(\rho=1)$ to largely opened containers $(\rho \gg 1)$.

One finally obtains the following expression for the mean flow field $\mathbf{F}$ :

$$
\begin{aligned}
& r<R: \quad \mathbf{F}_{i}=-\frac{\omega}{6} \frac{1}{R^{4}}\left[r\left(r^{2}+\beta R^{2}\right)\left(\begin{array}{c}
\cos (2 \theta) \\
-\sin (2 \theta)
\end{array}\right)\right. \\
&\left.+r^{3}\left(\begin{array}{c}
0 \\
-\sin (2 \theta)
\end{array}\right)\right]+o\left(\frac{a^{2}}{R}\right), \\
& r>R: \quad \mathbf{F}_{o}=-\frac{\omega}{12} \frac{R^{2}}{r^{3}}\left(\begin{array}{c}
\cos (2 \theta)\left[1-\frac{r^{4}}{R^{\prime 4}}\right] \\
\sin (2 \theta)\left[1+\frac{r^{4}}{R^{\prime 4}}\right]
\end{array}\right)+o\left(\frac{a^{2}}{R}\right) .
\end{aligned}
$$

Its streamlines, parametrized by $\lambda$, satisfy

$$
\begin{gathered}
r<R: \frac{r^{2}}{R^{2}}\left(\frac{r^{2}}{R^{2}}-\frac{R^{4}}{2 R^{\prime 4}}-\frac{1}{2}\right) \sin (2 \theta)=\lambda, \\
r>R: \frac{R^{2}}{2} \frac{R^{\prime 4}-r^{4}}{r^{2} R^{\prime 4}} \sin (2 \theta)=\lambda .
\end{gathered}
$$

The mean flow fields and the mean flow stream lines are sketched in Fig. 12 for closed containers $(\rho=1, \beta=-1)$ and in Fig. 13 for open containers $\left(\rho=2, \beta \approx-\frac{1}{2}\right)$. A focalization of mean flows on the line joining the foci is noticeable in closed containers but is largely weakened in open ones. This difference only traces back to the potential flow driven by $\beta$. We determine below its consequences on the phase field.

\section{Basic state of instability}

(a) The phase boundary conditions. Within the expansion (3), the condition $\mathbf{k} \cdot \mathbf{n}=0$ at the roll boundary can be written: $\sin \theta\left[1-3 a \cos ^{2}(\theta)\right]+O\left(a^{2}\right)=0$. It is always fulfilled at the central roll $(\theta=0)$, never at focus centers $(\theta= \pm \pi / 2)$ and never simultaneously on the whole boundary. Additional modes not taken into account in the present expansion of the phase field would thus be required to achieve it exactly. However, we emphasize that the status of this boundary condition is more phenomenological than analytical and, in particular, has not been addressed for the large curvatures encountered near the focus centers. We thus use it as a useful mean for estimating the curvature parameter $a$ by imposing almost perpendicular rolls for $\theta$ about $\pi / 4$. We then obtain $a=\frac{2}{3}+o(a)$. A value of order unity of $a$, although required to model satisfactorily the phase field, might appear incompatible with a perturbative expansion. Our guess is that the physical mechanism of pattern destabilization derived at weak curvature is sufficiently generic to operate at large ones. Then, applying our procedure for $a=\frac{2}{3}$ should be considered as a quantitative extrapolation of a qualitatively correct mechanism. This will be supported by the agreement between the corresponding solution and the experimental observations. Another phase boundary condition is in order at the locations of largest curvature $\chi$ : the focus centers. When foci are in equilibrium, the phase advection by mean flow, of order $O\left(a^{2} / R\right)$, balances the phase diffusion, of order $\chi$ $\left[k(0, \pm R)-k_{o}\right]$. Since $\chi$ is of $O(1)$ near a focus center, this gives $k(0, \pm R)-k_{o}=O\left(a^{2} / R\right)$ where $a=O(1)$ and $R$ $=O(10)$ in extended cells. We then obtain $k(0, \pm R)-k_{o}$ $=o\left(a^{2}\right)$ that expresses the wave-number selection by foci. Within the expansion (3), this yields $\Delta=-3 b-5 c$. 
(b) The basic state of instability. Introducing the mean flow field $\mathbf{F}$ found in Eqs. (14) and (15) into the phase equation (1) yields

$$
\begin{aligned}
\frac{\partial \varphi}{\partial t}= & k_{o} D_{\|}\left[\left(6 b-2 a \Delta-\beta \frac{\alpha \delta}{3}\right) \frac{y}{R^{2}}+\left(20 c-\frac{\alpha \delta}{3}\right) \frac{y^{3}}{R^{4}}\right. \\
& \left.+\left(10 a^{2}+6 d-\alpha \delta\right) \frac{y x^{2}}{R^{4}}\right]
\end{aligned}
$$

with

$$
\alpha=-\left[\frac{\gamma k A^{2} \tau}{\partial B / \partial k}\right]\left(k_{o}, \varepsilon, \operatorname{Pr}\right), \quad D_{\|}=-\left[\frac{1}{\tau} \frac{\partial(k B)}{\partial k}\right]\left(k_{o}, \varepsilon, \operatorname{Pr}\right) .
$$

As $(\partial B / \partial k)\left(k_{o}, \varepsilon, \operatorname{Pr}\right)$ is negative and $B\left(k_{o}, \varepsilon, \operatorname{Pr}\right)=0$, both variables $\alpha$ and $D_{\|}$are positive.

We notice that each mode of the phase equation (18) is actually involved in the phase-field expansion (3). This ensures the closure of the expansion of the $\mathrm{CN}$ equations and enables us to rewrite them as an algebraic dynamical system, by a mode to mode identification. Solving it in steady states gives the following determination of the basic states of instability at second order in $a$ :

$$
\begin{gathered}
{\left[b, c, d+\frac{5}{3} a^{2}, \Delta, \alpha \delta\right]=\Delta_{c}\left[\frac{2}{3} \beta, \frac{1}{5}, 2-(1+2 \beta), 12\right],} \\
\Delta_{c}=a^{2} \frac{\alpha}{2+\alpha(1+p)} .
\end{gathered}
$$

As assumed at the earliest stage, the compression parameters $b, c, d$, and $\Delta$ are second order in $a$. On the other hand, $\delta$ and therefore $\omega$ appear to be always positive. The constant sign of $\omega$ implies, from Eq. (14), that the direction of $\mathbf{F}$ is solely governed by $\beta$ in steady focus pairs. As expected, relation (20) then shows that the basic state of instability of focus pairs is parametrized $\beta$. We emphasize that this means that it actually depends on the kind of container.

\section{Local stability analysis}

We consider the local wave numbers displayed by steady focus pairs and investigate whether they belong to the Busse balloon. The minimal wave numbers of the phase field (3) are reached at the boundaries of the central roll: $(x, y)$ $=( \pm R, 0)$. They amount to $k( \pm R, 0)=k_{o}(1-a)+o\left(a^{2}\right)$ and may yield roll nucleation by a localized Eckhaus instability, actually observed experimentally [Fig. 6(a)].

The maximal wave number $k_{m}$ takes place on the $y$ axis. Here, $k$ reduces to the following expression:

$$
k(0, y)=k_{o}\left[1+\Delta_{c}\left\{\left(\frac{y^{2}}{R^{2}}+\beta\right)^{2}-(\beta+1)^{2}\right\}\right] .
$$

According to it, both the location and the value of $k_{m}$ depend on $\beta$.

(i) For $\beta=-\frac{1}{2}, k_{m}$ is reached at both focus centers $(x, y)$ $=(0,0)$ and the pattern center $(x, y)=(0,0)$. Then $\Delta=0$ and $k_{m}=k_{o}$.

(ii) For $\beta<-\frac{1}{2}, k_{m}$ is reached only at the pattern center $(x, y)=(0,0)$. Then $\Delta>0$ and $k_{m}=k_{o}(1+\Delta)$.

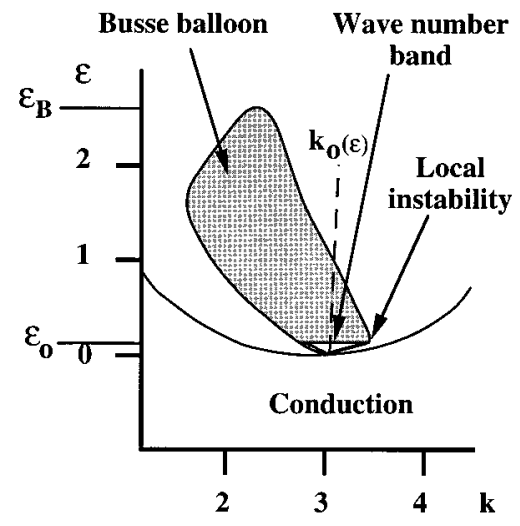

(a)

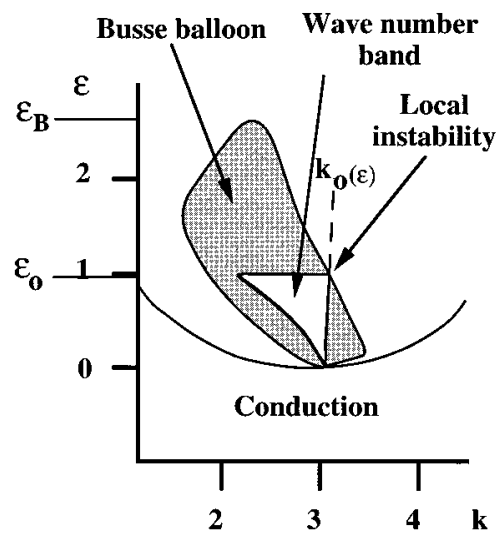

(b)

FIG. 14. Sketch of the diagram of stability of straight rolls, the Busse balloon, as a function of the Rayleigh number at a low Prandtl number $(\operatorname{Pr} \approx 1)$ (dashed domain). The wave number selected by foci, $k_{0}(\varepsilon)$, crosses the balloon at the "top," at values of $\varepsilon$ of the same order of magnitude as $\varepsilon_{B}$, the $\varepsilon$ limit of stable straight rolls. The local wave numbers of focus pairs are computed for $p=0$, $a=\frac{2}{3}$, and $\operatorname{Pr}=0.7$. (a) Closed containers: $\beta=-1$. Focus pairs display a wave-number band that crosses the Busse balloon at the "side." This induces a local instability at values of $\varepsilon, \varepsilon_{o}$, much smaller than $\varepsilon_{B}: \varepsilon_{o} / \varepsilon_{B}=O\left(10^{-1}\right)$. (b) Open containers: $\rho^{4} \gg 1$, $\beta=-\frac{1}{2}$. Crossing of the Busse balloon occurs near the "top," at values of $\varepsilon, \varepsilon_{o}$, of the order of its highest allowable value $\varepsilon_{B} / \varepsilon_{B}=O(1)$. Time dependence is thus inhibited inside the Busse balloon.

The first case is not dangerous since, at least for $\operatorname{Pr}>0.5$, $k_{o}$ lies well inside the Busse balloon up to large values of $\varepsilon$ [35]. On the opposite side, the second case may well yield a local instability at the pattern center for sufficiently large values of $\Delta$. Since $\Delta$ is proportional to $(1+2 \beta)$, this means that the local stability of focus pairs depends on the kind of container, as analyzed below.

In closed containers, $\rho=1, \beta=-1, \Delta=\Delta_{c}, \quad$ and $k_{m}=k_{o}\left(1+\Delta_{c}\right)$. At low Prandtl number $\operatorname{Pr} \approx 1$, Fig. 14(a) shows that $\Delta_{c}$ grows sufficiently fast with $\varepsilon$ to make $k_{m}$ cross the stability boundaries as soon as $\varepsilon \approx 0.1$. Focus pairs are then locally unstable well inside the Busse balloon.

As $\rho$ grows from $\rho=1$ (closed containers) to $\rho=\infty$ (open containers), $\beta$ increases from -1 to $-\frac{1}{2}$ and $\Delta$ decreases 
from $\Delta_{c}$ to 0 , whatever the Prandtl number. Meanwhile the maximal wave number is still reached at the pattern center but the roll compression decreases: $k_{m}=k_{o}(1+\Delta)$. This makes the threshold of local instability rise, as shown in Fig. 14(b). In particular, in the limit of widely opened containers, $\rho^{4} \gg 1, \Delta$ vanishes so that $k_{m}=k_{o}$, at any Prandtl number. No pinching can then occur until $k_{o}$ crosses the Busse balloon. At least for $\operatorname{Pr}>0.5$ [35], this prevents time dependence up to values of $\varepsilon$ of the order of its highest allowable value $\varepsilon_{B}$ $\left[\varepsilon_{o} \approx 1, \varepsilon_{B} \approx 2.5\right.$ in Fig. 14(b)]. Focus pairs are thus locally stable in the same $\varepsilon$ range as the Busse balloon.

In open containers, time dependence of focus pairs is thus inhibited inside the Busse balloon at least for any Prandtl number larger than 0.5 . This effect is hardly noticeable at high Prandtl number since, owing to the vanishing of mean flow sources, closed and open containers become physically equivalent, in particular regarding focus pair stability: $\operatorname{Pr} \rightarrow \infty, \gamma \rightarrow 0, \alpha \rightarrow 0, \Delta \rightarrow 0$, and $k_{m} \rightarrow k_{o}$, in either kind of containers. It is however spectacular at low Prandtl number, $\operatorname{Pr} \approx 1$, since focus pairs are much more unstable in closed containers than in open ones. In the latter, their time dependence is then surprisingly suppressed within the Busse balloon, not by removing mean flows as in the large Prandtl number limit, but by weakening their focalization on the pattern center.

\section{DISCUSSION}

We confront the experimental observations to the results of our analysis, following the above splitting between symmetric and asymmetric structures. We then focus attention on the role of geometry with respect to the mechanisms governing convective structures.

\section{A. Symmetric structures}

The instability displayed by straight rolls agrees with the skewed-varicose instability regarding both onset and form (Fig. 2). On the other hand, foci display a steady offcentering of focus singularities starting from $\varepsilon=0$ and growing with $\varepsilon$ [14-19] (Fig. 5). This does not fit with a largescale instability starting from a definite onset above the convective threshold. However the amplification of the offcentering indicates a loss of stiffness that recovers the main features brought about by the focus instability $[9,10]$.

Both these symmetric structures show, at most, very weak changes of their spatiotemporal behaviors in open containers. This agrees with the conclusions of our analysis of their stability: symmetric structures are (nearly) insensitive to mean flow boundary conditions.

\section{B. Asymmetric structures}

In open containers, the analysis of focus pairs predicts a weakening of the main dangerous mode: a focalization of the mean flow on the axis joining foci to the pattern center. Evidencing directly this effect is not an easy task, owing to the difficulties inherent to mean flow measurement or mean flow visualization [16], and we did not achieve it. Instead, we have checked its consequences by comparing our observations with the predictions regarding both the onset of time dependence and the wave-number field.

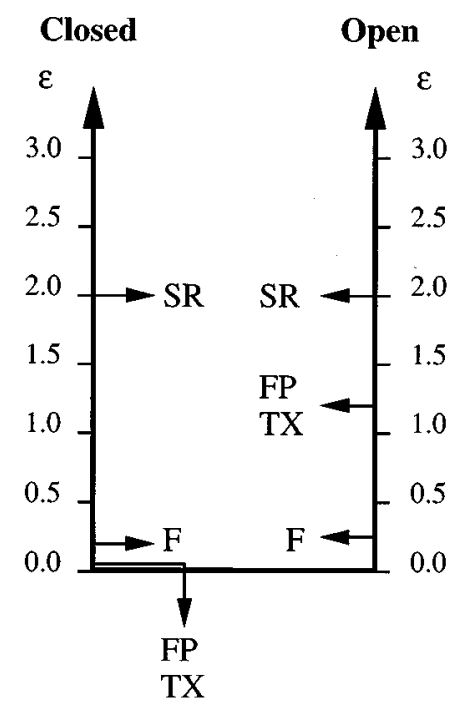

FIG. 15. Sketch of the threshold of time-dependence of various structures in closed and open containers. Straight rolls, foci, and focus pairs are labeled SR, F, and FP, respectively. Notice the sensitivity (independence) of focus pairs and textures (straight rolls and foci) to the type of container. Notice also the similarity of the threshold of time dependence of focus pairs and textures with that of foci in closed containers and that of straight rolls in open ones. Opening containers thus changes the status of asymmetric structures from that of most unstable structures to that of nearly most stable structures.

The main findings of our analysis are a delay of the onset of time dependence and an equality of the wave numbers at the pattern center and at the foci [Fig. 14(b)]. Both are nicely corroborated by our experiment (Fig. 7). However, for the sake of a better accuracy of this comparison, we take into account in Appendix D the perturbations induced by the annular sheet on the mean flow. It gives rise to an additional backflow on the line joining the focus to the pattern center. The maximal wave number is then reached at the pattern center only, so that local instability and defect nucleation should occur there, in agreement with Fig. 8. On the other hand, its value $k_{\mathrm{pc}}$ should be slightly larger than that displayed at the foci $k_{f}$, as confirmed by our data (Fig. 7).

After the first defect nucleation has occurred, patterns do not exhibit periodic dynamics as in closed containers [13] but restabilize in another focus pair involving one less roll pair [Fig. 6(c)]. A similar behavior may be observed on straight rolls as they encounter the skewed-varicose instability when increasing $\varepsilon$ (cf. Sec. III B and Refs. [17] and [29]). In the present case, restabilization may be understood by a slight dependence of the parameter $\alpha$ governing the local stability with respect to the mean wave number [see Eq. (19)]: removing one roll pair may then be sufficient to reduce $\alpha$ below the critical value at which defect nucleation is triggered [13]. In addition, since $\alpha$ is proportional to $\varepsilon$, the higher $\varepsilon$ is at the first defect nucleation, the larger the reduction of $\alpha$ may be and the better are the chances of observing restabilization. In agreement with this statement, experiments reveal that defect nucleation occurs too low in closed containers for allowing restabilization and sufficiently high in open containers for achieving it. The experimental features of focus pairs and especially their sensitivity to mean 
flow boundary conditions are thus well recovered from the $\mathrm{CN}$ equations.

\section{The role of symmetry}

Both experiment and analysis agree with a link between the sensitivity to mean flow boundary conditions and the symmetry of the wave-vector field. This correlation is sketched in Fig. 15 by drawing a comparison between the onset of time dependence of structures in the two kinds of containers. We address below its origin and its consequences by investigating the essential role of symmetry with respect to the route to time dependence.

\section{Continuous symmetry}

Continuous symmetry of the phase field precludes the existence of mean flows $(\mathbf{F}=\mathbf{0})$ and thus denies to mean flow boundary conditions any influence on symmetric states. These boundary conditions might however influence the symmetry-breaking instabilities, either the local ones or the large-scale ones.

In straight rolls, local instabilities are rejected since the wave number is selected: all rolls are unstable or none are. In foci, local unstable wave numbers might arise since wavenumber selection is only reached at large distance from the focus center. However, they would then be encountered on all azimuths so that the growth of instability could be compatible with the preservation of rotational symmetry. We note that such rotationally invariant dynamical states are actually observed as phase-traveling waves in simulations [36] and experiments $[19,32]$ in large aspect ratio containers. However, since they do not modify pattern geometry, they stand outside the scope of the problem addressed here.

Only large-scale instabilities can thus yield a time dependence of symmetric geometries. Although they involve mean flows, our analysis has shown that their onsets are independent on mean flow boundary conditions.

\section{Asymmetry}

In contrast with symmetric structures, asymmetric structures involve some mean flows, even in steady states: $\mathbf{F} \neq \mathbf{0}$. As shown on the model of asymmetric pattern, the focus pair, these flows raise phase gradients not only through their rotational part but also through their potential part. This results in a localization of pattern stress whose features depend on the mean pressure gradient and thus on mean flow boundary conditions.

Owing to the low magnitude of mean flows, the consequence of pattern stress might be thought to be negligible. They are however enhanced by the aspect ratio owing to the cumulative effect of mean flow stretch. At least at low Prandtl number and moderate aspect ratio, they then succeed in inducing local instability prior to large-scale instability.

\section{Correlation between symmetry and bifurcation}

The difference of behaviors of symmetric structures compared to asymmetric ones traces back to the vanishing of their mean flows $(\mathbf{F}=\mathbf{0})$. This important degeneracy inhibits the retroaction of large scales $(\mathbf{F})$ on small scales $(\mathbf{k})$ and prevents localization of pattern stress. This results in a modi- fication of both the route to time dependence (symmetry allows at most a slow evolution of wave number; asymmetry enhances phase gradients) and the bifurcation to instability (symmetry induces large-scale instability; asymmetry triggers local instability).

As confirmed by the different sensitivities to mean flow boundary conditions, the above distinctions especially indicate that symmetric and asymmetric structures are not physically equivalent (Fig. 15). Of course, this does not mean that the physical ingredients governing these structures are different (e.g., primarily instability, mean Reynolds stresses, flow incompressibility, etc.) but that the interplay between them generates different mechanisms and then different behaviors. Accordingly, asymmetries or distortions, whatever their magnitude, stand as essential modes of extended patterns.

A consequence of these statements is that, despite their apparent similarity, foci and focus pairs refer to different physical mechanisms. In particular, focus pairs cannot be viewed as the mere juxtaposition of two foci nor can an asymmetric or a distorted structure be analyzed in terms of symmetric structures. By contrast, the similarity between the behaviors of textures and focus pairs in both closed and open containers validates the latter as a good candidate for modeling textures. This suggests that, at least for moderate aspect ratios, focus pair might actually capture the essential mechanisms governing textures dynamics.

\section{CONCLUSION}

Convective structures are governed by only two hydrodynamical scales, the roll scale and the pattern scale. They thus provide a minimal model for studying scale interactions. We have been studying it by focusing on model structures involving simple geometries.

Although different on a number of points, model structures display such similar features in usual containers that one can hardly decide which of them accurately models textures. In order to improve their comparison, we have introduced a change of boundary condition by separating the mean flow boundary from the roll flow boundary. This made the boundary of the convective domain permeable to mean flows and therefore transformed the usual "closed" containers into "open", ones regarding these flows.

The change of container has been applied while keeping the same convective structures and thus the same mean flow sources. It has resulted in two opposite behaviors: straight rolls and axisymmetrical rolls (foci) kept the same behavior; focus pairs and textures displayed a spectacularly large delay of time dependence. The former structures involve a continuous symmetry of the wave-vector field and the latter structures none. Their respective sensitivity to the change of mean flow boundary condition has been recovered analytically by exploiting the consequences of the existence or of the failure of such symmetry. The origin of the sensitivity differences traces back to the degeneracy displayed in symmetric structures through the vanishing of mean flows. This actually decouples not only steady states but also their onset of instability from mean flow boundary conditions. On the opposite side, asymmetry generates mean flows that enhance phase gradients by cumulative roll stretch. The resulting localization of pattern stress then succeeds in triggering local insta- 
bility prior to large scale instability, at least in moderate aspect ratio cells and at low Prandtl number.

The opposite behaviors of symmetric and asymmetric structures show that their physics actually differ. In particular, textures cannot be modeled by foci but, to the present analysis, by focus pairs. This points to the physical mechanism governing focus pairs (see Sec. IV C) as a relevant prototype of those at work in textures, at least for the moderate aspect ratios addressed here.

The fact that our experiment has been performed at moderate aspect ratio raises some questions regarding pattern dynamics in larger cells. Then more substructures than in the present case would interact, some of them being cut by boundaries, the other being located in the bulk. The former structures are bound by impermeable walls and the latter by permeable ones regarding mean flows. Do they behave as in closed containers or rather as in open ones? Do they show the same dynamics or not? What is their respective sensitivity to a change of mean flow boundary condition? Answering these questions would improve our understanding of structures interactions and of the influence of boundary on the bulk dynamics.

Both our observations and analysis have finally revealed a link between the geometry and the dynamics of convective structures. According to it, distortion is a dangerous mode which, whatever its magnitude, modifies the route to time dependence by bringing about a coupling between large and small scales. This property traces back to the nonlocality generated in distorted states but inhibited in symmetric ones. Here, this nonlocality is provided by hydrodynamics. In other systems, other long-range interactions induced by electromagnetic fields or chemical mediators may play this role. Then, the present system might appear as a minimal model for the understanding of the inner mechanisms governing their organization or their dynamics.

\section{APPENDIX A: THE CROSS-NEWELL EQUATIONS}

The Cross-Newell equations describe the basic interactions between the phase field and the mean flow field [8]. They may be split into self and mutual interactions.

(i) Roll-roll interaction. Diffusive terms of the Boussinesq equations give rise to a local interaction between neighboring rolls. This results in an anisotropic diffusion of the roll position and thus of the phase field [6].

(ii) Roll action upon mean flows. It corresponds to the Siggia-Zippelius mechanism by which distorted rolls behave as local mean flow sources [26]. The resulting mean flow is linked to its sources in a nonlocal way.

(iii) Mean flow action upon rolls. It results from the advection of roll flows by the mean flow. Depending on the boundary conditions imposed on the phase field, it results either in phase drift, phase distortions, or both of them $[28,12]$. Whereas the link between phase advection and roll distortion is local, the one between mean flow and roll phase results from a spatial integration of the phase advection and is thus nonlocal.

(iv) Mean-flow-mean-flow interaction. It arises from the diffusion and the selfadvection of the mean flow. It reduces to vertical diffusion here (and thus, for a Poiseuille profile, to a multiplication by a constant) for the following reasons:
Since the horizontal spatial scale of the mean flow is large compared to the cell depth, horizontal diffusion may be neglected with respect to vertical diffusion. Length scales being nondimensionalized with the cell depth and time scales with the vertical diffusion time, the order of magnitude of mean flow self-advection, mean flow diffusion, and mean flow sources are, according to Eq. (2), $\operatorname{Pr}^{-1} R^{-1} \mathbf{F}^{2}, \mathbf{F}$, and $\operatorname{Pr}^{-1} R^{-1} \varepsilon^{2}$, respectively. Then, for $\operatorname{Pr}>1, \mathrm{R}>10$, and $\varepsilon<1$, the condition of equilibrium between the two former terms and the latter gives $\mathbf{F} \approx \operatorname{Pr}^{-1} R^{-1} \varepsilon^{2}$. The effective Reynolds number $\operatorname{Re}(\mathbf{F})$, i.e., the ratio of the self-advection to diffusion, is then, $\operatorname{Re}(\mathbf{F})=O\left(\operatorname{Pr}^{-2} R^{-2} \varepsilon^{2}\right)$. In the present case of extended cells $(R \gg 1)$ and in the vicinity of the convective threshold $(\varepsilon \ll 1)$, it is quite small so that the self-advection of F may be neglected compared to its diffusion.

When taken into account simultaneously, these interactions yield the Cross-Newell equations (1) and (2) where the first equation (1) is a phase-diffusion equation supplemented by an advection term of the phase by the mean flow and the second equation (2) expresses the Siggia-Zippelius mechanism.

\section{APPENDIX B: CONTINUITY OF THE PRESSURE FIELD AT THE INTERFACE}

The hydrodynamical interface $\left(R^{\prime}<r<R^{+}\right)$separates an inner zone $\left(r<R^{-}\right)$from an outer zone $\left(R^{+}<r<R^{\prime}\right)$ [Fig. 1(a)]. Two pressure fields $\Pi_{i}$ and $\Pi_{o}$ have been defined by Eqs. (11) and (12) in each of them. Our purpose is to evaluate the corresponding pressure drop $\delta \Pi$ across their interface:

$$
\delta \Pi=\Pi_{o}\left(R^{+}, \theta\right)-\Pi_{i}\left(R^{-}, \theta\right) .
$$

This will be performed first by determining a continuous matching of the pressure gradients $\nabla \Pi$ in the interface, second by evaluating its order of magnitude, and finally by deducing the corresponding pressure drop $\delta \Pi$.

\section{Continuous matching of the pressure gradients}

The mean flow $\mathbf{F}$ may be split in the whole system into a rotational part $\mathbf{R}$ and a potential part $\nabla \Pi$, both divergencefree:

$$
\mathbf{F}=\mathbf{R}+\nabla \Pi
$$

This splitting is not unique but examples are $\left(\mathbf{F}_{i r}, \boldsymbol{\nabla} \Pi_{i}\right)$ in the inner zone and $\left(\mathbf{F}_{o r}, \nabla \Pi_{o}\right)$ in the outer zone, as defined in Sec. IV C 3. Since $\mathbf{F}$ is continuous in the whole system, determining a continuous matching $\nabla \Pi$ of the pressure gradients $\nabla \Pi_{i}$ and $\nabla \Pi_{o}$ in the interface turns out to construct an explicit continuous expression of the rotational flow $\mathbf{R}$ in such a way that it corresponds to $\mathbf{F}_{i r}$ in the inner zone and $\mathbf{F}_{\text {or }}$ in the outer zone.

Let us label $R_{r}$ and $R_{\theta}$ its radial and orthoradial components and introduce the following coupling between mean flow sources and vertical vorticity:

$$
R_{r}=-\frac{r}{6} \chi \Omega_{z} \cot (2 \theta)
$$


where $\chi$ is a function of $r$, a priori undetermined. In order for the vertical vorticity of $\mathbf{R}$ to be equal to $\Omega_{z}$, the function $R_{\theta}$ must satisfy

$$
r R_{\theta}=\int_{0}^{r} r\left(1+\frac{\chi}{3}\right) \Omega_{z} d r
$$

In the convective domain, the choice $\chi=1$ yields $\mathbf{R}=\mathbf{F}_{i r}$. In the conductive domain, relation (B3) shows that the radial component $R_{r}$ of $\mathbf{R}$ is always zero since $\Omega_{z}$ vanishes in the outer zone. Its orthoradial component $R_{\theta}$ can be forced to vanish too, by choosing $\chi$ in the interface so that $R_{\theta}\left(R_{+}, \theta\right)=0$. Then, in the outer zone, $\mathbf{R}=\mathbf{F}_{o r}=\mathbf{0}$.

Different choices of $\chi$ satisfying the above requirements may be made inside the interface. The relevance of this degree of freedom is ensured by the fact that their differences correspond to potential flows whose pressure drop (the circulation of the corresponding flow between the two sides of the interface) vanishes, owing to (B3) and (B4).

\section{Order of magnitude of the pressure gradient in the interface}

We seek to deduce the order of magnitude of $\nabla \Pi$ from those of $\mathbf{F}$ and $\mathbf{R}$ in the interface. At first, we assume that the interface does not increase the order of magnitude of the mean flow in the inner zone and outer zone. On the contrary, the roll compression would be larger than usual so that the threshold of time dependence would be much smaller than in closed containers. This scenario is rejected by experimental observations (see Sec. III D 2). Then $\mathbf{F}$ is still $O\left(a^{2} / R\right)$ on both sides of the interface so that its shear rate is $O\left(a^{2} / R\right)$.

Since the spatial derivatives are $O(1)$ inside the interface, the order of magnitude of $\mathbf{F}$ and $\mathbf{R}$ inside it are the same as that of their vertical vorticity $\Omega_{z I}$. This vorticity results from three different phenomena: roll distortion, mean flow shear, and roll amplitude variations. The first two contribute to $\Omega_{z I}$ to the same order of magnitude $a^{2} / R$. The contribution of the last may be easily estimated by emphasizing that rolls end normally to the interface. Then, locally, they look like a set of parallel rolls fading away a normal boundary. For reasons of symmetry, the mean flow that they produce by amplitude decay must then be parallel to their axis and invariant by translation along the boundary so that its vertical vorticity vanishes. Put together, these estimates yield $\Omega_{z I}, \mathbf{F}, \mathbf{R}$, and $\nabla \Pi$ to be at least $O\left(a^{2} / R\right)$ in the interface.

\section{Pressure drop across the interface}

Since the pressure gradient $\nabla \Pi$ is well defined and continuous in the interface, evaluating the pressure drop $\delta \Pi$ across it makes sense. Its order of magnitude is that of the pressure gradient, $O\left(a^{2} / R\right)$, multiplied by the interface width, $O(1)$ in the present problem, so that $\delta \Pi=O\left(a^{2} / R\right)$. Since, in an extended cell, $R^{-1}=O\left(10^{-1}\right)=o(1)$, we finally obtain $\delta \Pi=o\left(a^{2}\right)$, so that the pressure fields $\Pi_{o}$ and $\Pi_{i}$ match continuously at second order in a:

$$
\Pi_{o}(R, \theta)-\Pi_{i}(R, \theta)=o\left(a^{2}\right) .
$$

\section{APPENDIX C: INTERFACE VORTICITY AND MEAN FLOW SHEAR}

In an open container, the net vertical vorticity in the upper-right quarter $D_{o}$ of the cell $\left(0<r<R^{\prime}, 0<\theta<\pi / 2\right)$ may be deduced from relations (4) $-(6)$ :

$$
\iint_{D_{o}} \Omega_{z} d S=\frac{\omega}{4}+\omega_{I}
$$

Since $\omega_{I}=-\omega / 3$, the interface vorticity dominates the vertical vorticity produced in the bulk and imposes a negative sign of the net vertical vorticity in the domain $D_{o}$. The circulation of the mean flow field $\mathbf{F}$ along the boundaries of $D_{o}$ must then be negative [Fig. 13(a)]. This is in contrast with the case of closed containers, where the analogous circulation along the upper-right quarter $D_{c}(0<r<R, 0<\theta$ $<\pi / 2)$ is positive [Fig. 12(a)].

At some places of the boundary of $D_{o}$, the mean flow must therefore point in a direction opposite to that displayed in a closed container. Figure 13 shows that this mainly occurs in the outer zone. In the remaining parts, especially in the inner zone, the amplitude of the mean flow, and in particular the back flow, is reduced, but the direction is kept.

The mean flow shear at the interface results from the large variations of the orthoradial component $\mathbf{F} \cdot \mathbf{e}_{\theta}$ across the interface. Their origin may be understood as follows. Mass conservation implies continuity of $\mathbf{F} \cdot \mathbf{r}$ across the interface. Equating the circulation of the mean flow on an infinitesimal contour in the interface to the flux of vertical vorticity then yields

$$
\left[\mathbf{F}\left(R_{+}\right)-\mathbf{F}\left(R_{-}\right)\right] e_{\theta}=\frac{\omega_{I}}{R}
$$

so that the shear is directly produced by the interface vorticity $\omega_{I}$. Its magnitude is $O\left(a^{2} / R\right)$ since $\omega_{I}=O(\omega)=O\left(a^{2}\right)$. It is thus of the same order as the mean flow $\mathbf{F}$ and modifies it considerably, as shown by the comparison between Figs. 12 and 13.

The interface vorticity thus produces local effects (shear at the interface) but also important nonlocal effects (mean flow direction, decrease of the back flow) of primary importance for the transition to time dependence.

\section{APPENDIX D: INFLUENCE OF THE SHEET ON MEAN FLOWS}

Apart from local vorticity sources produced at the roll boundaries by roll amplitude decay, the sheet induces a viscous shear in the conductive domain that decreases the flow driven by pressure gradients [Fig. 1(c)]. This additional effect may be easily evaluated by assuming a Poiseuille profile for the mean flow. This results in the following relation between the potential mean flows in the inner and outer zones $\mathbf{F}_{i p}$ and $\mathbf{F}_{o p}$ and the pressure field $\Pi$ :

$$
\mathbf{F}_{i p}=\nabla \Pi, \quad \mathbf{F}_{o p}=t \nabla \Pi, \quad t=1-3 \delta(1-\delta),
$$

where $\delta=d_{1} / d$ is the relative distance of the sheet to a horizontal plate and $t$ a transmission factor. 
The sheet perturbation may thus be handled by renormalizing the pressure fields in both the inner and outer zones by $\Pi_{i}=\Pi, \Pi_{o}=t \Pi$, respectively. Since, the actual pressure field $\Pi$ is continuous (see Appendix B), this means introducing a virtual pressure drop at the interface $\Pi_{o}-\Pi_{i}=-(1$ $-t) \Pi_{i}$. The remaining boundary conditions of the system being unchanged, the basic state of instability can be found straightforwardly as in Sec. IV C 4. Only the value of $\beta$ is modified:

$$
\beta=-\frac{1+\rho^{-4}}{2-(1-t)\left(1-\rho^{-4}\right)} .
$$

In the limit $\rho \gg 1, \beta$ simplifies to $\beta=-1 /(1+t)$ which, for $t<1$, is smaller than the value $-\frac{1}{2}$ expected without sheet perturbation $(t=1)$. This indicates the presence of an additional back flow on the line joining focus and pattern center, which increases roll compression and lowers the onset of time dependence. Since $\beta<-\frac{1}{2}$, the maximum wave number is reached at the pattern center so that a localized instability should first occur there. Its value is

$$
k(0,0)=k_{o}\left(1+\frac{1-t}{1+t} \Delta_{c}\right) .
$$

As expected, $t=1$ corresponds to fully open containers $\left[k(0,0)=k_{o}\right]$, and $t=0$ to closed containers $[k(0,0)$ $\left.=k_{o}\left(1+\Delta_{c}\right)\right]$.

In the present experiment, $\delta=\frac{1}{4}$ and $\rho=2$, so that $t=\frac{7}{16}$ and $\beta=-0.72$. Taking for $k_{o}$ the expression determined close to onset of convection given by Manneville and Piquemal [35] and recalling that $\Delta_{c}=a^{2} \alpha /[2+\alpha(1+p)]$ with $\alpha=4.19 \varepsilon$ at $\operatorname{Pr}=0.71, p \approx 0$, and $a^{2} \approx \frac{1}{2}[9,10]$, we obtain, for the wave number at the pattern center, the curve plotted in Fig. 7.
[1] M. C. Cross and P. C. Hohenberg, Rev. Mod. Phys. 65, 851 (1993).

[2] P. Manneville, Dissipative Structures and Weak Turbulence (Academic, San Diego, CA, 1990).

[3] A. C. Newell, T. Passot, and J. Lega, Annu. Rev. Fluid Mech. 25, 399 (1993).

[4] F. H. Busse, Rep. Prog. Phys. 41, 1929 (1978); R. M. Clever and F. H. Busse, J. Fluid Mech. 91, 319 (1979); 102, 61 (1981).

[5] A. C. Newell and J. A. Whitehead, J. Fluid Mech. 38, 279 (1969); L. A. Segel, ibid. 38, 203 (1969).

[6] Y. Pomeau and P. Manneville, J. Phys. Lett. 40, 609 (1979).

[7] Y. Pomeau and P. Manneville, J. Phys. Lett. 42, 1067 (1981).

[8] M. C. Cross and A. C. Newell, Physica D 10, 299 (1984).

[9] A. C. Newell, in Propagation in Systems Far from Equilibrium, edited by J. E. Wesfreid, H. Brand, P. Manneville, G. Albinet, and N. Boccara (Springer-Verlag, Berlin, 1988), p. 122.

[10] A. C. Newell, T. Passot, and M. Souli, J. Fluid Mech. 220, 187 (1990).

[11] A. Pocheau, J. Phys. (France) 49, 1127 (1988).

[12] A. Pocheau, in New Trends in Nonlinear Dynamics and Pattern Forming Phenomena: The Geometry of Non Equilibrium, Vol. 237 of NATO Advanced Study Institute, Series B: Physics, edited by P. Huerre and P. Coullet (Plenum, New York, 1988).

[13] A. Pocheau, J. Phys. (France) 50, 2059 (1989).

[14] V. Croquette and A. Pocheau, in Cellular Structures and Instabilities, edited by J. E. Wesfreid and S. Zaleski, Lecture Note in Physics (Springer-Verlag, New York, 1983).

[15] G. Ahlers, D. S. Cannell, and V. Steinberg, Phys. Rev. Lett. 54, 1373 (1985); V. Steinberg, G. Ahlers, and D. S. Cannell, Phys. Scr. 9, 97 (1985).

[16] V. Croquette, P. Le Gal, A. Pocheau, and R. Guglielmetti, Europhys. Lett. 1, 393 (1986).

[17] V. Croquette, Contemp. Phys. 30, 153 (1989).
[18] F. Daviaud, Ph.D. thesis, Université Paris VI, 1990 (unpublished).

[19] Y. Hu, R. E. Ecke, and G. Ahlers, Phys. Rev. E 48, 4399 (1993).

[20] G. Ahlers and R. P. Behringer, Phys. Rev. Lett. 40, 712 (1978); R. P. Behringer and G. Ahlers, J. Fluid Mech. 125, 219 (1982).

[21] A. Pocheau, V. Croquette, and P. Le Gal, Phys. Rev. Lett. 55, 1094 (1985).

[22] H. S. Greenside, M. C. Cross, and W. M. Coughran, Jr., Phys. Rev. Lett. 60, 2269 (1988).

[23] J. P. Gollub, A. R. McCarriar, and J. F. Steinmann, J. Fluid Mech. 125, 259 (1982).

[24] M. S. Heutmaker and J. P. Gollub, Phys. Rev. A 35, 242 (1987).

[25] F. Daviaud and A. Pocheau, Europhys. Lett. 9, 675 (1989).

[26] E. D. Siggia and A. Zippelius, Phys. Rev. Lett. 47, 835 (1981); A. Zippelius and E. Siggia, Phys. Fluids 26, 2905 (1983).

[27] M. C. Cross, Phys. Rev. A 27, 490 (1983).

[28] A. Pocheau, V. Croquette, P. Le Gal, and C. Poitou, Europhys. Lett. 8, 915 (1987).

[29] P. Kolodner, R. W. Walden, A. Passner, and C. M. Surko, J. Fluid Mech. 163, 195 (1986).

[30] R. P. Behringer, Rev. Mod. Phys. 57, 657 (1985); H. Gao and R. P. Behringer, Phys. Rev. A 30, 2837 (1984).

[31] A. Pocheau, Ph.D. thesis, Université Paris VII, 1987 (unpublished).

[32] Y. Hu, R. E. Ecke, and G. Ahlers, Phys. Rev. Lett. 72, 2191 (1994).

[33] M. Assenheimer and V. Steinberg, Phys. Rev. Lett. 70t, 3888 (1993).

[34] S. W. Morris, E. Bodenschatz, D. S. Cannell, and G. Ahlers, Phys. Rev. Lett. 71, 2026 (1993); Y. Hu, R. E. Ecke, and G. Ahlers, ibid. 74, 391 (1995).

[35] P. Manneville and J. M. Piquemal, Phys. Rev. A 28, 1774 (1983).

[36] L. Tuckerman and D. Barkley, Phys. Rev. Lett. 61, 408 (1980). 